\title{
Palladium-Catalyzed C-H Activation/Intramolecular Amination Reaction: A New Route to 3-Aryl/Alkylindazoles
}

\author{
Kiyofumi Inamoto,* Mika Katsuno, Tadataka Saito, Takao Sakamoto, Kou Hiroya* \\ Graduate School of Pharmaceutical Sciences, Tohoku University \\ 6-3, Aoba, Aramaki, Aoba-ku, Sendai, 980-8578, Japan
}

Supporting Information

\section{General}

All reactions were carried out under Ar atmosphere unless otherwise noted. Ethanol was distilled from sodium under Ar atmosphere. Dimethyl sulfoxide (DMSO) was distilled from $\mathrm{CaH}_{2}$ under $\mathrm{Ar}$ atmosphere. Melting points were measured with a Yazawa micro melting point apparatus and uncorrected. IR spectra were recorded on a SHIMADZU FTIR-8400. ${ }^{1}$ H-NMR spectra were recorded on JEOL JNM-AL400 (400 MHz) or JNM-ECA600 (600 $\mathrm{MHz}$ ) spectrometer using tetramethylsilane (TMS) as an internal standard. Chemical shifts $(\delta)$ are given from TMS (0 ppm) and coupling constants are expressed in Herts $(\mathrm{Hz})$. The following abbreviations are used: $\mathrm{s}=$ singlet, $\mathrm{d}=$ doublet, $\mathrm{t}=$ triplet, $\mathrm{q}=$ quartet, quint $=$ quintet, sep $=$ septet, $\mathrm{dd}=$ double doublet, $\mathrm{dt}=$ double triplet, $\mathrm{td}=$ triple doublet, $\mathrm{ddd}=$ double double doublet, $\mathrm{m}=$ multiplet, br.s $=$ broad singlet, and $\mathrm{br}=$ broad signal. ${ }^{13} \mathrm{C}-\mathrm{NMR}$ spectra were recorded on JEOL JNM-AL400 (100 MHz) or JNM-ECA600 (150 MHz) spectrometer and chemical shifts $(\delta)$ are given from ${ }^{13} \mathrm{CDCl}_{3}(77.0 \mathrm{ppm})$ and ${ }^{13} \mathrm{C}_{6} \mathrm{D}_{6}(128.0$ ppm). Mass spectra and high resolution mass spectra were measured on JEOL JMS-DX303 and MS-AX500 instruments, respectively. FAB-mass spectra was measured on JEOL JMS-700 instrument. Elemental analyses were performed by Yanaco CHN CORDER MT-6. 


\section{Preparation of Starting Materials}

\section{General Procedure for the Preparation of Hydrazones}

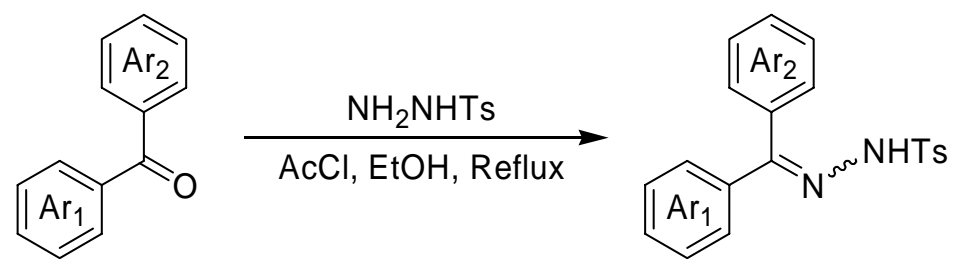

Acetyl chloride was slowly added to ethanol $(1 \mathrm{~mL})$ at $0^{\circ} \mathrm{C}$ and stirred for $30 \mathrm{~min}$ at the same temperature. The reaction mixture was warmed to room temperature and stirred for another $30 \mathrm{~min}$. The resulting mixture and a ethanol solution of the ketone were added to a ethanol solution of 4-methylphenylsulfonylhydrazide, then heated under reflux. The reaction mixture was treated with saturated aqueous $\mathrm{NaHCO}_{3}$ and extracted with ethyl acetate. The combined organic layer was washed with brine and dried over $\mathrm{MgSO}_{4}$. The solvent was evaporated and the residue was purified by silica gel column chromatography.

\section{Benzophenone 4-Methylphenylsulfonylhydrazone (1)}

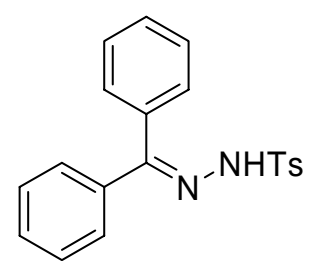

According to the general procedure, a mixture of benzophenone (1.5 g, $8.2 \mathrm{mmol})$, acetyl chloride (1.3 g, $16.5 \mathrm{mmol})$, and 4-methylphenylsulfonylhydrazide (3.6 g, $19.8 \mathrm{mmol})$ in ethanol $(40 \mathrm{~mL})$ was heated under reflux for $40 \mathrm{~h}$. The crude material was purified by silica gel column chromatography [hexane-ethyl acetate (4:1)] to afford 1 (2.4 g, 82\%) as a colorless solid.

$\mathrm{mp} 182-183^{\circ} \mathrm{C}$ (colorless prisms from hexane-acetone, lit. ${ }^{1)} \mathrm{mp} 184-185^{\circ} \mathrm{C}$ );

IR $v$ (film, $\mathrm{cm}^{-1}$ ) 3209, 1169, 772, 694, 667;

${ }^{1} \mathrm{H}-\mathrm{NMR}\left(400 \mathrm{MHz}, \mathrm{CDCl}_{3}\right) \delta 2.43(3 \mathrm{H}, \mathrm{s}), 7.10-7.13(2 \mathrm{H}, \mathrm{m}), 7.25-7.34(5 \mathrm{H}, \mathrm{m}), 7.44$ $(2 \mathrm{H}, \mathrm{d}, J=6.8 \mathrm{~Hz}), 7.50-7.54(4 \mathrm{H}, \mathrm{m}), 7.85(2 \mathrm{H}, \mathrm{d}, J=8.4 \mathrm{~Hz})$;

${ }^{13} \mathrm{C}-\mathrm{NMR}\left(100 \mathrm{MHz}, \mathrm{CDCl}_{3}\right) \delta 21.7,127.5,127.8,128.1,128.2,129.6,129.7,129.8,130.0$, 
131.0, 135.4, 136.3, 144.0, 154.1;

MS $m / z 350\left(\mathrm{M}^{+}, 23.4\right), 195$ (100);

HRMS calcd for $\mathrm{C}_{20} \mathrm{H}_{18} \mathrm{~N}_{2} \mathrm{O}_{2} \mathrm{~S} 350.1089$, found 350.1081 .

\section{4,4'-Dimethoxybenzophenone 4-Methylphenylsulfonylhydrazone (3a)}<smiles>COc1ccc(C(=NN)c2ccc(OC)cc2)cc1</smiles>

According to the general procedure, a mixture of 4,4'-dimethoxybenzophenone $(0.16 \mathrm{~g}$, $0.66 \mathrm{mmol})$, acetyl chloride $(0.25 \mathrm{~g}, 3.3 \mathrm{mmol})$, and 4-methylphenylsulfonylhydrazide $(0.37 \mathrm{~g}, 2.0 \mathrm{mmol})$ in ethanol $(7 \mathrm{~mL})$ was heated under reflux for $40 \mathrm{~h}$. The crude material was purified by silica gel column chromatography [hexane-ethyl acetate (2:1)] to afford 3a $(0.19 \mathrm{~g}, 69 \%)$ as a light brown solid.

$\mathrm{mp} 138-140^{\circ} \mathrm{C}$ (light brown plates from hexane-ethyl acetate, lit. ${ }^{2)} \mathrm{mp} 136-137^{\circ} \mathrm{C}$ );

IR $v$ (film, $\left.\mathrm{cm}^{-1}\right)$ 3200, 1252, 1161, 837, 662;

${ }^{1} \mathrm{H}-\mathrm{NMR}\left(400 \mathrm{MHz}, \mathrm{CDCl}_{3}\right) \delta 2.42(3 \mathrm{H}, \mathrm{s}), 3.79(3 \mathrm{H}, \mathrm{s}), 3.87(3 \mathrm{H}, \mathrm{s}), 6.79(2 \mathrm{H}, \mathrm{d}, J=8.8$ $\mathrm{Hz}), 7.00(2 \mathrm{H}, \mathrm{d}, J=8.0 \mathrm{~Hz}), 7.05(2 \mathrm{H}, \mathrm{d}, J=8.8 \mathrm{~Hz}), 7.32(2 \mathrm{H}, \mathrm{d}, J=8.8 \mathrm{~Hz}), 7.37(2 \mathrm{H}, \mathrm{d}$, $J=8.8 \mathrm{~Hz}), 7.50(1 \mathrm{H}, \mathrm{s}), 7.84(2 \mathrm{H}, \mathrm{d}, J=8.0 \mathrm{~Hz})$;

${ }^{13} \mathrm{C}-\mathrm{NMR}\left(100 \mathrm{MHz}, \mathrm{CDCl}_{3}\right) \delta 21.7,55.3,55.4,113.5,115.0,123.0,127.8,129.1,129.47$, $129.50,129.8,135.5,143.9,154.2,160.5,160.9$;

MS $m / z 410\left(\mathrm{M}^{+}, 14.5\right), 255(100)$;

HRMS calcd for $\mathrm{C}_{22} \mathrm{H}_{22} \mathrm{~N}_{2} \mathrm{O}_{4} \mathrm{~S} 410.1301$, found 410.1301 .

\section{4,4'-Dimethylbenzophenone 4-Methylphenylsulfonylhydrazone (3b)}<smiles>Cc1ccc(C(=NNS)c2ccc(C)cc2)cc1</smiles> 
According to the general procedure, a mixture of 4,4'-dimethylbenzophenone $(32.3 \mathrm{mg}$, $0.15 \mathrm{mmol}$ ), acetyl chloride (60.3 $\mathrm{mg}, 0.77 \mathrm{mmol})$, 4-methylphenylsulfonylhydrazide $(85.8$ $\mathrm{mg}, 0.46 \mathrm{mmol})$, and $\mathrm{MgSO}_{4}(63.5 \mathrm{mg}, 0.53 \mathrm{mmol})$ in ethanol $(4 \mathrm{~mL})$ was heated under reflux for $45 \mathrm{~min}$. The crude material was purified by silica gel column chromatography [hexane-ethyl acetate (9:1)] to afford $\mathbf{3 b}(43.4 \mathrm{~g}, 75 \%)$ as a colorless solid.

$\mathrm{mp} 150-152^{\circ} \mathrm{C}$ (colorless prisms from hexane-ethyl acetate);

IR $v$ (film, $\left.\mathrm{cm}^{-1}\right)$ 3202, 1171, 824, 667;

${ }^{1} \mathrm{H}-\mathrm{NMR}\left(400 \mathrm{MHz}, \mathrm{CDCl}_{3}\right) \sigma 2.33(3 \mathrm{H}, \mathrm{s}), 2.42(6 \mathrm{H}, \mathrm{s}), 6.97(2 \mathrm{H}, \mathrm{d}, J=8.0 \mathrm{~Hz}), 7.08(2 \mathrm{H}$, $\mathrm{d}, J=8.0 \mathrm{~Hz}), 7.29-7.34(6 \mathrm{H}, \mathrm{m}), 7.50(1 \mathrm{H}, \mathrm{s}), 7.83(2 \mathrm{H}, \mathrm{d}, J=8.4 \mathrm{~Hz})$;

${ }^{13} \mathrm{C}-\mathrm{NMR}\left(100 \mathrm{MHz}, \mathrm{CDCl}_{3}\right) \delta 21.3,21.4,21.7,127.5,127.8,128.1,128.8,129.5,130.3$, $133.8,135.5,139.9,140.0,143.9,154.6$;

MS $m / z 378\left(\mathrm{M}^{+}, 16.6\right), 223$ (100);

HRMS calcd for $\mathrm{C}_{22} \mathrm{H}_{22} \mathrm{~N}_{2} \mathrm{O}_{2} \mathrm{~S} 378.1402$, found 378.1405 .

\section{3,3'-Dimethoxybenzophenone 4-Methylphenylsulfonylhydrazone (3c)}<smiles>COc1cccc(C(=NNSc2ccccc2)c2cccc(OC)c2)c1</smiles>

According to the general procedure, a mixture of 3,3'-dimethoxybenzophenone $(0.12 \mathrm{~g}$, $0.52 \mathrm{mmol})$, acetyl chloride $(0.20 \mathrm{~g}, 2.6 \mathrm{mmol})$, and 4-methylphenylsulfonylhydrazide $(0.19 \mathrm{~g}, 1.0 \mathrm{mmol})$ in ethanol $(5 \mathrm{~mL})$ was heated under reflux for $1 \mathrm{~h}$. The crude material was purified by silica gel column chromatography [hexane-ethyl acetate (4:1)] to afford 3c $(0.17 \mathrm{~g}, 80 \%)$ as a colorless solid.

$\mathrm{mp} 103-105^{\circ} \mathrm{C}$ (colorless plates from hexane-ethyl acetate);

IR $v$ (film, $\mathrm{cm}^{-1}$ ) 3198, 1169, 872, 667;

${ }^{1} \mathrm{H}-\mathrm{NMR}\left(400 \mathrm{MHz}, \mathrm{CDCl}_{3}\right) \delta 2.42(3 \mathrm{H}, \mathrm{s}), 3.77(3 \mathrm{H}, \mathrm{s}), 3.79(3 \mathrm{H}, \mathrm{s}), 6.60(1 \mathrm{H}, \mathrm{d}, J=2.4$ $\mathrm{Hz}), 6.65(1 \mathrm{H}, \mathrm{d}, J=8.0 \mathrm{~Hz}), 6.88(1 \mathrm{H}, \mathrm{dd}, J=8.0,2.4 \mathrm{~Hz}), 6.97(1 \mathrm{H}, \mathrm{d}, J=8.0 \mathrm{~Hz}), 7.01$ $(1 \mathrm{H}, \mathrm{dd}, J=8.0,2.4 \mathrm{~Hz}), 7.07(1 \mathrm{H}, \mathrm{t}, J=2.4 \mathrm{~Hz}), 7.18(1 \mathrm{H}, \mathrm{t}, J=8.0 \mathrm{~Hz}), 7.32(2 \mathrm{H}, \mathrm{d}, J=$ 
$8.2 \mathrm{~Hz}), 7.41(1 \mathrm{H}, \mathrm{t}, J=8.0 \mathrm{~Hz}), 7.58(1 \mathrm{H}, \mathrm{s}), 7.85(2 \mathrm{H}, \mathrm{d}, J=8.2 \mathrm{~Hz})$;

${ }^{13} \mathrm{C}-\mathrm{NMR}\left(100 \mathrm{MHz}, \mathrm{CDCl}_{3}\right) \delta 21.6,55.2,55.4,112.6,113.5,115.6,115.7,120.1,120.4$, $127.9,129.1,129.6,131.0,132.3,135.5,137.6,144.1,153.8,159.4,160.5$;

MS $m / z 410\left(\mathrm{M}^{+}, 6.4\right), 255(100)$;

HRMS calcd for $\mathrm{C}_{22} \mathrm{H}_{22} \mathrm{~N}_{2} \mathrm{O}_{4} \mathrm{~S} 410.1301$, found 410.1293 .

3-Methoxy-3'-nitrobenzophenone 4-Methylphenylsulfonylhydrazone (3d)<smiles>CCNN=C(c1cccc(OC)c1)c1cccc([N+](=O)[O-])c1</smiles>

3-Methoxyphenylmagnesium bromide ( $0.77 \mathrm{M}$ solution in THF, $1.9 \mathrm{~mL}, 1.5 \mathrm{mmol})$ was added to a solution of 3-nitrobenzaldehyde $(0.20 \mathrm{~g}, 1.3 \mathrm{mmol})$ in THF $(7 \mathrm{~mL})$ at $-78^{\circ} \mathrm{C}$ and stirred for $35 \mathrm{~min}$ at the same temperature. Saturated aqueous $\mathrm{NH}_{4} \mathrm{Cl}(5 \mathrm{~mL})$ was added to the reaction mixture and the aqueous phase was extracted with ethyl acetate $(10 \mathrm{~mL} \times 3)$. The combined organic layer was washed with water $(5 \mathrm{~mL})$ and brine $(5 \mathrm{~mL})$, and dried over $\mathrm{MgSO}_{4}$. The solvent was evaporated and the residue was purified by silica gel column chromatography [hexane-ethyl acetate $(4: 1)$ ] to give 3-methoxy- $\alpha$-(3-nitrophenyl)benzyl alcohol (0.33 g, 95\%) as a colorless oil.

${ }^{1} \mathrm{H}-\mathrm{NMR}\left(400 \mathrm{MHz}, \mathrm{CDCl}_{3}\right) \delta 2.68(1 \mathrm{H}, \mathrm{br}), 3.78(3 \mathrm{H}, \mathrm{s}), 5.86(1 \mathrm{H}, \mathrm{s}), 6.82-6.84(1 \mathrm{H}, \mathrm{m})$, 6.91-6.93 (2H, m), 7.24-7.29 (1H, m), 7.45-7.51 (1H, m), $7.69(1 \mathrm{H}, \mathrm{d}, J=6.4 \mathrm{~Hz}), 8.09$ $(1 \mathrm{H}, \mathrm{d}, J=8.4 \mathrm{~Hz}), 8.27(1 \mathrm{H}, \mathrm{s})$;

${ }^{13} \mathrm{C}-\mathrm{NMR}\left(100 \mathrm{MHz}, \mathrm{CDCl}_{3}\right) \delta 55.3,75.2,112.2,113.5,118.8,121.2,122.4,129.3,129.9$, $132.3,144.3,145.6,148.2,159.8$.

A mixture of the above alcohol $(0.29 \mathrm{~g}, 1.1 \mathrm{mmol})$ and activated $\mathrm{MnO}_{2}(1.5 \mathrm{~g}, 17.3$ $\mathrm{mmol})$ in dichloromethane $(10 \mathrm{~mL})$ was stirred for $30 \mathrm{~min}$ at room temperature. The reaction mixture was diluted with methanol and filtered through a pad of Celite ${ }^{\circledR}$. The filtrate was evaporated under reduced pressure and the residue was purified by silica gel column chromatography [hexane-ethyl acetate (4:1)] to give 
3-methoxy-3'-nitrobenzophenone (0.29 g, 99\%).

${ }^{1} \mathrm{H}-\mathrm{NMR}\left(400 \mathrm{MHz}, \mathrm{CDCl}_{3}\right) \delta 3.88(3 \mathrm{H}, \mathrm{s}), 7.19(1 \mathrm{H}, \mathrm{dd}, J=8.0,2.6 \mathrm{~Hz}), 7.31(1 \mathrm{H}, \mathrm{d}, J=$ $8.0 \mathrm{~Hz}), 7.36(1 \mathrm{H}, \mathrm{s}), 7.43(1 \mathrm{H}, \mathrm{t}, J=8.0 \mathrm{~Hz}), 7.70(1 \mathrm{H}, \mathrm{t}, J=8.0 \mathrm{~Hz}), 8.14(1 \mathrm{H}, \mathrm{d}, J=8.0$ $\mathrm{Hz}), 8.44$ (1H, m), $8.63(1 \mathrm{H}, \mathrm{s})$;

${ }^{13} \mathrm{C}-\mathrm{NMR}\left(100 \mathrm{MHz}, \mathrm{CDCl}_{3}\right) \delta 55.3,114.1,119.3,122.4,124.3,126.3,129.3,135.1,137.2$, $138.7,147.7,159.5,193.4$.

According to the general procedure, a mixture of 3-methoxy-3'-nitrobenzophenone $(0.28$ g, $1.1 \mathrm{mmol})$, acetyl chloride $(0.43 \mathrm{~g}, 5.4 \mathrm{mmol})$, and 4-methylphenylsulfonylhydrazide $(0.40 \mathrm{~g}, 2.2 \mathrm{mmol})$ in ethanol $(6 \mathrm{~mL})$ was heated under reflux for $1.5 \mathrm{~h}$. The crude material was purified by silica gel column chromatography [hexane-ethyl acetate (4:1)] to afford 3d (0.24 g, including impurities), which was further purified by recrystallization from hexane-ethyl acetate to afford $3 \mathbf{d}$ as a single isomer in a pure form $(0.23 \mathrm{~g}, 49 \%)$.

$\mathrm{mp} 193-194^{\circ} \mathrm{C}$ (colorless prisms from hexane-ethyl acetate); IR $v\left(\right.$ film, $\left.\mathrm{cm}^{-1}\right)$ 3200, 1529, 1348, 1169, 814, 746, 687, 664;

${ }^{1} \mathrm{H}-\mathrm{NMR}\left(400 \mathrm{MHz}, \mathrm{CDCl}_{3}\right) \delta 2.45(3 \mathrm{H}, \mathrm{s}), 3.83(3 \mathrm{H}, \mathrm{s}), 6.64(1 \mathrm{H}, \mathrm{s}), 6.69(1 \mathrm{H}, \mathrm{d}, J=8.0$ $\mathrm{Hz}), 7.08(1 \mathrm{H}, \mathrm{dd}, J=8.0,2.6 \mathrm{~Hz}), 7.37(2 \mathrm{H}, \mathrm{d}, J=8.2 \mathrm{~Hz}), 7.475(1 \mathrm{H}, \mathrm{t}, J=8.0 \mathrm{~Hz})$, $7.480(1 \mathrm{H}, \mathrm{t}, J=8.0 \mathrm{~Hz}), 7.74(1 \mathrm{H}, \mathrm{s}), 7.81(1 \mathrm{H}, \mathrm{d}, J=8.0 \mathrm{~Hz}), 7.88(2 \mathrm{H}, \mathrm{d}, J=8.2 \mathrm{~Hz})$, $8.18(1 \mathrm{H}, \mathrm{d}, J=8.0 \mathrm{~Hz}), 8.23(1 \mathrm{H}, \mathrm{s})$;

${ }^{13} \mathrm{C}-\mathrm{NMR}\left(100 \mathrm{MHz}, \mathrm{CDCl}_{3}\right) \delta 21.7,55.5,113.5,116.0,119.9,122.2,124.2,127.9,129.2$, $129.7,130.9,131.4,132.9,135.0,138.1,144.5,148.2,151.1,160.7$;

MS m/z 425 (M+, 0.81), 270 (96.1), 269 (100), 224 (53.1);

HRMS calcd for $\mathrm{C}_{21} \mathrm{H}_{19} \mathrm{~N}_{3} \mathrm{O}_{5} \mathrm{~S} 425.1046$, found 425.1061;

Anal. calcd for $\mathrm{C}_{21} \mathrm{H}_{19} \mathrm{~N}_{3} \mathrm{O}_{5} \mathrm{~S}: \mathrm{C}, 59.28 ; \mathrm{H}, 4.50 ; \mathrm{N}, 9.88$. found: C, 59.12; H, 4.40; N, 9.68.

\section{3'-Cyano-3-methoxybenzophenone 4-Methylphenylsulfonylhydrazone (3e)}<smiles>CCCCCCCNN=C(c1cccc(C#N)c1)c1cccc(OC)c1</smiles> 
3-Methoxyphenylmagnesium bromide ( $0.60 \mathrm{M}$ solution in THF, $11 \mathrm{~mL}, 6.6 \mathrm{mmol})$ was added to a solution of 3-bromobenzaldehyde $(0.94 \mathrm{~g}, 5.1 \mathrm{mmol})$ in THF $(20 \mathrm{~mL})$ at $0^{\circ} \mathrm{C}$ and stirred for $15 \mathrm{~min}$ at room temperature. Saturated aqueous $\mathrm{NH}_{4} \mathrm{Cl}(10 \mathrm{~mL})$ was added to the reaction mixture and the aqueous phase was extracted with ethyl acetate $(10 \mathrm{~mL} \times 3)$. The combined organic solution was washed with water $(10 \mathrm{~mL})$ and brine $(10 \mathrm{~mL})$, and dried over $\mathrm{MgSO}_{4}$. The solvent was evaporated and the residue was purified by silica gel column chromatography [hexane-ethyl acetate (9:1)] to give 3-bromo- $\alpha$-(3-methoxyphenyl)benzyl alcohol (1.5 g, quant.) as a colorless oil.

${ }^{1} \mathrm{H}-\mathrm{NMR}\left(400 \mathrm{MHz}, \mathrm{CDCl}_{3}\right) \delta 3.62(1 \mathrm{H}, \mathrm{s}), 3.66(3 \mathrm{H}, \mathrm{s}), 5.55(1 \mathrm{H}, \mathrm{s}), 6.74(1 \mathrm{H}, \mathrm{td}, J=8.0$, $2.1 \mathrm{~Hz}), 6.81-6.84(2 \mathrm{H}, \mathrm{m}), 7.07(1 \mathrm{H}, \mathrm{t}, J=8.0 \mathrm{~Hz}), 7.14-7.19(2 \mathrm{H}, \mathrm{m}), 7.30(1 \mathrm{H}, \mathrm{d}, J=8.0$ $\mathrm{Hz}), 7.47(1 \mathrm{H}, \mathrm{s})$.

A mixture of the above alcohol $(1.5 \mathrm{~g}, 5.2 \mathrm{mmol})$ and activated $\mathrm{MnO}_{2}(13.0 \mathrm{~g}, 149.5$ $\mathrm{mmol})$ in dichloromethane $(30 \mathrm{~mL})$ was stirred for $1 \mathrm{~h}$ at room temperature. The reaction mixture was diluted with methanol and filtered through a pad of Celite ${ }^{\circledR}$. The filtrate was evaporated under reduced pressure and the residue was purified by silica gel column chromatography [hexane-ethyl acetate (9:1)] to give 3-bromo-3'-methoxybenzophenone $(1.3 \mathrm{~g}, 86 \%)$ as a colorless solid.

${ }^{1} \mathrm{H}-\mathrm{NMR}\left(400 \mathrm{MHz}, \mathrm{CDCl}_{3}\right) \delta 3.83(3 \mathrm{H}, \mathrm{s}), 7.12(1 \mathrm{H}, \mathrm{dd}, J=8.2,2.6 \mathrm{~Hz}), 7.27-7.38(4 \mathrm{H}$, m), 7.66-7.69 (2H, m), $7.92(1 \mathrm{H}, \mathrm{s})$;

${ }^{13} \mathrm{C}-\mathrm{NMR}\left(100 \mathrm{MHz}, \mathrm{CDCl}_{3}\right) \delta 55.4,114.1,119.0,122.4,122.6,128.3,129.2,129.6,132.5$, $135.0,137.9,139.2,159.4,194.5$.

A mixture of the above benzophenone $(0.52 \mathrm{~g}, 1.8 \mathrm{mmol}), \mathrm{Pd}_{2}(\mathrm{dba})_{3}(97.2 \mathrm{mg}, 0.11$ $\mathrm{mmol})$, dppf (0.24 g, $0.43 \mathrm{mmol}), \mathrm{CuCN}(0.63 \mathrm{~g}, 7.1 \mathrm{mmol})$, and $\mathrm{Et}_{4} \mathrm{NCN}(0.28 \mathrm{~g}, 1.8$ $\mathrm{mmol})$ in dioxane $(10 \mathrm{~mL})$ was heated under reflux for $4.5 \mathrm{~h}$. The reaction mixture was filtered through a pad of Celite ${ }^{\circledR}$ and the filtrate was washed with saturated aqueous $\mathrm{NaHCO}_{3}\left(10 \mathrm{~mL}\right.$ x 3) and brine $(10 \mathrm{~mL}$ x 1$)$, and dried over $\mathrm{MgSO}_{4}$. The solvent was evaporated and the residue was purified by silica gel column chromatography [hexane-ethyl acetate (4:1)] to give 3'-cyano-3-methoxybenzophenone $(0.41 \mathrm{~g}, 98 \%)$ as a yellow solid. 
IR $v\left(\right.$ film, $\left.\mathrm{cm}^{-1}\right)$ 2232, 1663, 1288, 1246, 745;

${ }^{1} \mathrm{H}-\mathrm{NMR}\left(400 \mathrm{MHz}, \mathrm{CDCl}_{3}\right) \delta 3.86(3 \mathrm{H}, \mathrm{s}), 7.18(1 \mathrm{H}, \mathrm{ddd}, J=8.0,2.4,1.0 \mathrm{~Hz}), 7.28(1 \mathrm{H}$, $\mathrm{dt}, J=8.0,1.0 \mathrm{~Hz}), 7.33(1 \mathrm{H}, \mathrm{m}), 7.41(1 \mathrm{H}, \mathrm{t}, J=8.0 \mathrm{~Hz}), 7.63(1 \mathrm{H}, \mathrm{t}, J=7.8 \mathrm{~Hz}), 7.86$ $(1 \mathrm{H}, \mathrm{dt}, J=7.8,1.4 \mathrm{~Hz}), 8.03(1 \mathrm{H}, \mathrm{dt}, J=7.8,1.4 \mathrm{~Hz}), 8.07(1 \mathrm{H}, \mathrm{t}, J=1.4 \mathrm{~Hz})$;

${ }^{13} \mathrm{C}-\mathrm{NMR}\left(100 \mathrm{MHz}, \mathrm{CDCl}_{3}\right) \delta 55.1,112.3,113.9,117.5,118.9,122.2,128.9,129.1,132.8$, 133.3, 134.8, 137.0, 138.0, 159.2, 193.4;

$\mathrm{MS} m / z 237\left(\mathrm{M}^{+}, 85.9\right), 135$ (100);

HRMS calcd for $\mathrm{C}_{15} \mathrm{H}_{11} \mathrm{NO}_{2} 237.0790$, found 237.0774.

According to the general procedure, a mixture of 3'-cyano-3-methoxybenzophenone $(0.13 \mathrm{~g}, \quad 0.55 \mathrm{mmol})$, acetyl chloride $(0.22 \mathrm{~g}, \quad 2.7 \mathrm{mmol})$, and 4-methylphenylsulfonylhydrazide $(0.20 \mathrm{~g}, 1.1 \mathrm{mmol})$ in ethanol $(5 \mathrm{~mL})$ was heated under reflux for $75 \mathrm{~min}$. The crude material was purified by silica gel column chromatography [hexane-ethyl acetate (4:1)] to afford $3 \mathbf{e}$ as a mixture of two isomers $(0.21 \mathrm{~g}, 94 \%)$. The ratio of two isomers was determined by ${ }^{1} \mathrm{H}-\mathrm{NMR}$ spectrum (major isomer : minor isomer = $7.7: 1$ ), and recrystallization gave the single isomer (major isomer).

major isomer: $\mathrm{mp} 174-175^{\circ} \mathrm{C}$ (colorless scales from hexane-ethyl acetate);

IR $v$ (film, $\mathrm{cm}^{-1}$ ) 3236, 2226, 1165, 907, 868, 806, 669;

${ }^{1} \mathrm{H}-\mathrm{NMR}\left(400 \mathrm{MHz}, \mathrm{CDCl}_{3}\right) \delta 2.45(3 \mathrm{H}, \mathrm{s}), 3.83(3 \mathrm{H}, \mathrm{s}), 6.60(1 \mathrm{H}, \mathrm{s}), 6.66(1 \mathrm{H}, \mathrm{d}, J=8.0$ $\mathrm{Hz}), 7.07(1 \mathrm{H}, \mathrm{dd}, J=8.0,2.4 \mathrm{~Hz}), 7.36(2 \mathrm{H}, \mathrm{d}, J=8.2 \mathrm{~Hz}), 7.41(1 \mathrm{H}, \mathrm{t}, J=8.0 \mathrm{~Hz}), 7.47$ $(1 \mathrm{H}, \mathrm{t}, J=8.0 \mathrm{~Hz}), 7.60(1 \mathrm{H}, \mathrm{d}, J=8.0 \mathrm{~Hz}), 7.66(1 \mathrm{H}, \mathrm{s}), 7.72-7.74(2 \mathrm{H}, \mathrm{m}), 7.85(2 \mathrm{H}, \mathrm{d}, J$ $=8.2 \mathrm{~Hz})$;

${ }^{13} \mathrm{C}-\mathrm{NMR}\left(100 \mathrm{MHz}, \mathrm{CDCl}_{3}\right) \delta 21.7,55.5,112.6,113.5,116.0,118.3,119.8,127.8,129.1$, 129.7, 130.9, 130.98, 130.99, 131.2, 131.4, 132.8, 137.4, 144.4, 151.2, 160.7;

MS $m / z 405\left(\mathrm{M}^{+}, 2.0\right), 250$ (100), 249 (90.9);

HRMS calcd for $\mathrm{C}_{22} \mathrm{H}_{19} \mathrm{~N}_{3} \mathrm{O}_{3} \mathrm{~S} 405.1147$, found 405.1138;

Anal. calcd for $\mathrm{C}_{22} \mathrm{H}_{19} \mathrm{~N}_{3} \mathrm{O}_{3} \mathrm{~S}: \mathrm{C}, 65.17 ; \mathrm{H}, 4.72 ; \mathrm{N}, 10.36$. found: C, 65.19; H, 4.84; N, 10.34 . 
3'-Ethoxycarbonyl-3-methoxybenzophenone 4-Methylphenylsulfonylhydrazone (3f)<smiles>CCCCNN=C(c1cccc(OC)c1)c1cccc(C(=O)OCC)c1</smiles>

A mixture of 3-bromo-3'-methoxybenzophenone (0.74 g, $2.5 \mathrm{mmol}), \mathrm{Pd}_{2}(\mathrm{dba})_{3}(0.11 \mathrm{~g}$, $0.51 \mathrm{mmol})$, dppp (0.31 g, $0.76 \mathrm{mmol}), \mathrm{Et}_{3} \mathrm{~N}(4 \mathrm{~mL})$, and $\mathrm{EtOH}(4 \mathrm{~mL})$ in DMSO $(8 \mathrm{~mL})$ was heated at $70^{\circ} \mathrm{C}$ for $44 \mathrm{~h}$. The reaction mixture was filtered through a pad of Celite ${ }^{\circledR}$ and the filtrate was washed with $\mathrm{H}_{2} \mathrm{O}(10 \mathrm{~mL} \times 1)$, saturated aqueous $\mathrm{NaHCO}_{3}(10 \mathrm{~mL} \times 1), 3$ $\mathrm{N} \mathrm{HCl}(10 \mathrm{~mL} \times 1)$, and brine $(10 \mathrm{~mL} \times 1)$, and dried over $\mathrm{MgSO}_{4}$. The solvent was evaporated and the residue was purified by silica gel column chromatography [hexane-ethyl acetate (19:1 to 4:1)] to give 3'-ethoxycarbonyl-3-methoxybenzophenone $(0.41 \mathrm{~g}, 98 \%)$ as a yellow solid.

According to the general procedure, a mixture of 3'-ethoxycarbonyl-3-methoxybenzophenone ( $0.30 \mathrm{~g}, 1.0 \mathrm{mmol})$, acetyl chloride $(0.41 \mathrm{~g}, 5.2$ $\mathrm{mmol})$, and 4-methylphenylsulfonylhydrazide (0.39 g, $2.1 \mathrm{mmol})$ in ethanol $(10 \mathrm{~mL})$ was heated under reflux for $1 \mathrm{~h}$. The crude material was purified by silica gel column chromatography [hexane-ethyl acetate $(4: 1)$ ] to afford $3 \mathbf{f}$ as a mixture of two isomers (0.21 g, 94\%). The ratio of two isomers was determined by ${ }^{1} \mathrm{H}-\mathrm{NMR}$ spectrum (major isomer : minor isomer $=33: 1$ ), and recrystallization gave the single isomer (major isomer).

major isomer: $\mathrm{mp} 180-182^{\circ} \mathrm{C}$ (colorless prisms from hexane-ethyl acetate);

IR $v$ (film, $\mathrm{cm}^{-1}$ ) 3186, 1717, 1267, 1169;

${ }^{1} \mathrm{H}-\mathrm{NMR}\left(400 \mathrm{MHz}, \mathrm{CDCl}_{3}\right) \delta 1.40(3 \mathrm{H}, \mathrm{t}, J=7.2 \mathrm{~Hz}), 2.44(3 \mathrm{H}, \mathrm{s}), 3.78(3 \mathrm{H}, \mathrm{s}), 4.39(2 \mathrm{H}$, q, $J=7.2 \mathrm{~Hz}), 6.88-6.90(2 \mathrm{H}, \mathrm{m}), 7.02(1 \mathrm{H}, \mathrm{s}), 7.19(1 \mathrm{H}, \mathrm{t}, J=8.0 \mathrm{~Hz}), 7.33-7.35(3 \mathrm{H}, \mathrm{m})$, $7.44(1 \mathrm{H}, \mathrm{s}), 7.61(1 \mathrm{H}, \mathrm{t}, J=8.0 \mathrm{~Hz}), 7.79(1 \mathrm{H}, \mathrm{s}), 7.86(2 \mathrm{H}, \mathrm{d}, J=8.0 \mathrm{~Hz}), 8.18(1 \mathrm{H}, \mathrm{d}, J=$ $8.0 \mathrm{~Hz})$;

${ }^{13} \mathrm{C}-\mathrm{NMR}\left(100 \mathrm{MHz}, \mathrm{CDCl}_{3}\right) \delta 14.4,21.7,55.3,61.5,112.6,115.7,120.4,127.9,129.2$, 129.3, 129.6, 129.9, 131.1, 131.4, 132.1, 132.5, 135.2, 137.4, 144.2, 152.8, 159.4, 165.3; MS $m / z 452\left(\mathrm{M}^{+}, 4.5\right), 269(100)$; 
HRMS calcd for $\mathrm{C}_{24} \mathrm{H}_{24} \mathrm{~N}_{2} \mathrm{O}_{5} \mathrm{~S} 452.1406$, found 452.1408;

Anal. calcd for $\mathrm{C}_{24} \mathrm{H}_{24} \mathrm{~N}_{2} \mathrm{O}_{5} \mathrm{~S}$ : C, 63.70; H, 5.35; N, 6.19. found: C, 63.73; H, 5.38; N, 6.16.

\section{3-Hydroxybenzophenone 4-Methylphenylsulfonylhydrazone (3g)}<smiles>NN=C(c1ccccc1)c1cccc(O)c1</smiles>

According to the general procedure, a mixture of 3-hydroxybenzophenone ( $0.36 \mathrm{~g}, 1.8$ mmol), acetyl chloride $(0.71 \mathrm{~g}, 9.0 \mathrm{mmol})$, and 4-methylphenylsulfonylhydrazide $(0.67 \mathrm{~g}$, $3.6 \mathrm{mmol})$ in ethanol $(15 \mathrm{~mL})$ was heated under reflux for $8 \mathrm{~h}$. The crude material was purified by silica gel column chromatography [hexane-ethyl acetate $(2: 1)]$ to afford $\mathbf{3 g}$ (0.75 g, including impurities), which was further purified by recrystallization from hexane-ethyl acetate to afford $3 \mathrm{~g}(0.39 \mathrm{~g}, 60 \%)$ as an inseparable mixture of two isomers. The ratio of two isomers was determined by HPLC as $2.4: 1$.

IR $v\left(\right.$ film, $\left.\mathrm{cm}^{-1}\right) 3437,3179,1321,1161,839,772,704,669$;

${ }^{1} \mathrm{H}-\mathrm{NMR} /{ }^{13} \mathrm{C}-\mathrm{NMR}$ : only a ${ }^{1} \mathrm{H}-\mathrm{NMR}$ spectrum of $E$-/Z-mixture was obtained;

MS $m / z 366\left(\mathrm{M}^{+}, 10.6\right), 211$ (100);

HRMS calcd for $\mathrm{C}_{20} \mathrm{H}_{18} \mathrm{~N}_{2} \mathrm{O}_{3} \mathrm{~S} 366.1038$, found 366.1034;

Anal. calcd for $\mathrm{C}_{20} \mathrm{H}_{18} \mathrm{~N}_{2} \mathrm{O}_{3} \mathrm{~S}: \mathrm{C}, 65.55 ; \mathrm{H}, 4.95 ; \mathrm{N}, 7.64$. found: C, 65.30; H, 5.04; N, 7.70.

\section{3-Aminobenzophenone 4-Methylphenylsulfonylhydrazone (3h)}<smiles>NN=C(c1ccccc1)c1cccc(N)c1</smiles>

According to the general procedure, a mixture of 3-aminobenzophenone $(0.20 \mathrm{~g}, 1.0$ mmol), acetyl chloride $(0.40 \mathrm{~g}, 5.1 \mathrm{mmol})$, and 4-methylphenylsulfonylhydrazide $(0.38 \mathrm{~g}$, 
$2.0 \mathrm{mmol})$ in ethanol $(5 \mathrm{~mL})$ was heated under reflux for $45 \mathrm{~min}$. The crude material was purified by silica gel column chromatography [hexane-ethyl acetate $(2: 1)$ ] to afford $\mathbf{3 h}$ $(0.20 \mathrm{~g}, 54 \%)$ as an inseparable mixture of two isomers. The ratio of two isomers was determined by HPLC as $1.7: 1$.

IR $v$ (film, $\mathrm{cm}^{-1}$ ) 3381, 3215, 1167, 754, 704, 667;

${ }^{1} \mathrm{H}-\mathrm{NMR} /{ }^{13} \mathrm{C}-\mathrm{NMR}$ : only a ${ }^{1} \mathrm{H}-\mathrm{NMR}$ spectrum of $E$-/Z-mixture was obtained;

MS $m / z 365\left(\mathrm{M}^{+}, 5.4\right), 210(100)$;

HRMS calcd for $\mathrm{C}_{20} \mathrm{H}_{19} \mathrm{~N}_{3} \mathrm{O}_{2} \mathrm{~S} 365.1198$, found 365.1199;

Anal. calcd for $\mathrm{C}_{20} \mathrm{H}_{19} \mathrm{~N}_{3} \mathrm{O}_{2} \mathrm{~S}: \mathrm{C}, 65.73 ; \mathrm{H}, 5.24 ; \mathrm{N}, 11.50$. found: C, 65.51; H, 5.04; N, 11.41 .

3-Methoxybenzophenone 4-Methylphenylsulfonylhydrazone (3i)<smiles>COc1cccc(C(=NN)c2ccccc2)c1</smiles>

According to the general procedure, a mixture of 3-methoxybenzophenone $(0.29 \mathrm{~g}, 1.4$ mmol), acetyl chloride $(0.54 \mathrm{~g}, 6.9 \mathrm{mmol})$, and 4-methylphenylsulfonylhydrazide $(0.51 \mathrm{~g}$, $2.8 \mathrm{mmol})$ in ethanol $(10 \mathrm{~mL})$ was heated under reflux for $45 \mathrm{~min}$. The crude material was purified by silica gel column chromatography [hexane-ethyl acetate (4:1)] to afford $\mathbf{3 i}$ as a mixture of two isomers $(0.47 \mathrm{~g}, 90 \%)$. The ratio of two isomers was determined by ${ }^{1} \mathrm{H}-\mathrm{NMR}$ spectrum (major isomer : minor isomer $=1.3: 1$ ), and each isomer was obtained by recrystallization from hexane-diethyl ether.

major isomer: $\mathrm{mp} 156-158^{\circ} \mathrm{C}$ (colorless needles from hexane-diethyl ether, lit. ${ }^{3)} \mathrm{mp} 157^{\circ} \mathrm{C}$ ); IR $v$ (film, $\mathrm{cm}^{-1}$ ) 3198, 1169, 814, 694, 669;

${ }^{1} \mathrm{H}-\mathrm{NMR}\left(400 \mathrm{MHz}, \mathrm{CDCl}_{3}\right) \delta 2.43(3 \mathrm{H}, \mathrm{s}), 3.80(3 \mathrm{H}, \mathrm{s}), 6.61(1 \mathrm{H}, \mathrm{m}), 6.66(1 \mathrm{H}, \mathrm{dt}, J=$ 7.6, $1.0 \mathrm{~Hz}), 7.02(1 \mathrm{H}, \mathrm{ddd}, J=8.4,2.6,1.0 \mathrm{~Hz}), 7.25-7.34(6 \mathrm{H}, \mathrm{m}), 7.43(1 \mathrm{H}, \mathrm{t}, J=8.0$ $\mathrm{Hz}), 7.46(1 \mathrm{H}, \mathrm{m}), 7.57(1 \mathrm{H}, \mathrm{s}), 7.85(2 \mathrm{H}, \mathrm{d}, J=8.4 \mathrm{~Hz})$;

${ }^{13} \mathrm{C}-\mathrm{NMR}\left(100 \mathrm{MHz}, \mathrm{CDCl}_{3}\right) \delta 21.7,55.4,113.4,115.6,120.0,127.4,127.8,128.1,129.6$, 
$129.8,130.9,132.2,135.4,136.1,144.0,153.9,160.4$

MS $m / z 380\left(\mathrm{M}^{+}, 5.9\right), 225(100)$;

HRMS calcd for $\mathrm{C}_{21} \mathrm{H}_{20} \mathrm{~N}_{2} \mathrm{O}_{3} \mathrm{~S} 380.1194$, found 380.1204 .

minor isomer: $\mathrm{mp} 122-123^{\circ} \mathrm{C}$ (colorless prisms from hexane-diethyl ether);

IR $v$ (film, $\left.\mathrm{cm}^{-1}\right)$ 3200, 1169, 816, 789, 702, 665;

${ }^{1} \mathrm{H}-\mathrm{NMR}\left(400 \mathrm{MHz}, \mathrm{CDCl}_{3}\right) \delta 2.43(3 \mathrm{H}, \mathrm{s}), 3.77(3 \mathrm{H}, \mathrm{s}), 6.88(1 \mathrm{H}, \mathrm{dd}, J=8.0,2.4 \mathrm{~Hz})$, $6.94(1 \mathrm{H}, \mathrm{dd}, J=7.6,0.8 \mathrm{~Hz}), 7.05(1 \mathrm{H}, \mathrm{s}), 7.10-7.12(2 \mathrm{H}, \mathrm{m}), 7.18(1 \mathrm{H}, \mathrm{t}, J=8.2 \mathrm{~Hz})$, $7.32(2 \mathrm{H}, \mathrm{d}, J=8.4 \mathrm{~Hz}), 7.49-7.52(4 \mathrm{H}, \mathrm{m}), 7.85(2 \mathrm{H}, \mathrm{d}, J=8.4 \mathrm{~Hz})$;

${ }^{13} \mathrm{C}-\mathrm{NMR}\left(100 \mathrm{MHz}, \mathrm{CDCl}_{3}\right) \delta 21.7,55.3,112.6,115.6,120.4,127.9,128.2,129.1,129.6$, 129.7, 130.0, 131.0, 135.4, 137.7, 144.1, 153.9, 159.3;

MS $m / z 380\left(\mathrm{M}^{+}, 9.1\right), 225(100)$;

HRMS calcd for $\mathrm{C}_{21} \mathrm{H}_{20} \mathrm{~N}_{2} \mathrm{O}_{3} \mathrm{~S} 380.1195$, found 380.1189 .

\section{3-Chlorobenzophenone 4-Methylphenylsulfonylhydrazone (3j)}<smiles>CCNN=C(c1ccccc1)c1cccc(Cl)c1</smiles>

According to the general procedure, a mixture of 3-chlorobenzophenone $(0.19 \mathrm{~g}, 0.86$ $\mathrm{mmol})$, acetyl chloride $(0.34 \mathrm{~g}, 4.3 \mathrm{mmol})$, and 4-methylphenylsulfonylhydrazide $(0.32 \mathrm{~g}$, $1.7 \mathrm{mmol})$ in ethanol $(10 \mathrm{~mL})$ was heated under reflux for $10.5 \mathrm{~h}$. The crude material was purified by silica gel column chromatography [hexane-ethyl acetate (4:1)] to afford $3 \mathbf{j}(0.25$ $\mathrm{g}, 76 \%$ ) as an inseparable mixture of two isomers. The ratio of two isomers was determined by ${ }^{1} \mathrm{H}-\mathrm{NMR}$ spectrum (major isomer : minor isomer $=2: 1$ ).

IR $v\left(\right.$ film, $\left.\mathrm{cm}^{-1}\right)$ 3200, 1167, 885, 779, 760, 694, 665;

${ }^{1} \mathrm{H}-\mathrm{NMR} /{ }^{13} \mathrm{C}-\mathrm{NMR}$ : only a ${ }^{1} \mathrm{H}-\mathrm{NMR}$ spectrum of $E$-/Z-mixture was obtained;

MS $m / z 386\left(\mathrm{M}^{+}+2,8.2\right), 384\left(\mathrm{M}^{+}, 19.6\right), 229(100)$;

HRMS calcd for $\mathrm{C}_{20} \mathrm{H}_{17}{ }^{35} \mathrm{ClN}_{2} \mathrm{O}_{2} \mathrm{~S} 384.0699$, found 384.0694 .

Anal. calcd for $\mathrm{C}_{20} \mathrm{H}_{17} \mathrm{ClN}_{2} \mathrm{O}_{2} \mathrm{~S}: \mathrm{C}, 62.41 ; \mathrm{H}, 4.45 ; \mathrm{N}, 7.28$. found: $\mathrm{C}, 62.57 ; \mathrm{H}, 4.47 ; \mathrm{N}$, 


\section{3-Bromobenzophenone 4-Methylphenylsulfonylhydrazone (3k)}<smiles>NN=C(c1ccccc1)c1cccc(Br)c1</smiles>

According to the general procedure, a mixture of 3-bromobenzophenone (0.58 g, 2.2 mmol), acetyl chloride ( $0.87 \mathrm{~g}, 11.0 \mathrm{mmol})$, and 4-methylphenylsulfonylhydrazide ( $0.82 \mathrm{~g}$, $4.4 \mathrm{mmol})$ in ethanol $(15 \mathrm{~mL})$ was heated under reflux for $1.5 \mathrm{~h}$. The crude material was purified by silica gel column chromatography [hexane-ethyl acetate (4:1)] to afford $3 \mathbf{k}$ $(0.61 \mathrm{~g}, 64 \%)$ as a mixture of two isomers. Recrystallization gave the single isomer (major isomer).

major isomer: $\mathrm{mp} 198-200^{\circ} \mathrm{C}$ (colorless prisms from hexane-ethyl acetate);

IR $v\left(\right.$ film, $\left.\mathrm{cm}^{-1}\right)$ 3190, 1169, 795, 775, 760, 667;

${ }^{1} \mathrm{H}-\mathrm{NMR}\left(400 \mathrm{MHz}, \mathrm{CDCl}_{3}\right) \delta 2.44(3 \mathrm{H}, \mathrm{s}), 7.10(1 \mathrm{H}, \mathrm{d}, J=7.6 \mathrm{~Hz}), 7.24(2 \mathrm{H}, \mathrm{d}, J=8.0$ $\mathrm{Hz}), 7.28-7.37(4 \mathrm{H}, \mathrm{m}), 7.39-7.43(3 \mathrm{H}, \mathrm{m}), 7.46(1 \mathrm{H}, \mathrm{s}), 7.66(1 \mathrm{H}, \mathrm{d}, J=8.0 \mathrm{~Hz}), 7.86(2 \mathrm{H}$, $\mathrm{d}, J=8.0 \mathrm{~Hz})$;

${ }^{13} \mathrm{C}-\mathrm{NMR}\left(100 \mathrm{MHz}, \mathrm{CDCl}_{3}\right) \delta 21.7,123.9,126.8,127.4,127.9,128.2,129.6,130.0,131.0$, 131.2, 133.1, 133.2, 135.2, 135.8, 144.2, 152.4;

MS $m / z 430\left(\mathrm{M}^{+}+2,8.1\right), 428\left(\mathrm{M}^{+}, 7.8\right), 165(100)$;

HRMS calcd for $\mathrm{C}_{20} \mathrm{H}_{17}{ }^{81} \mathrm{BrN}_{2} \mathrm{O}_{2} \mathrm{~S} 430.0173$, found 430.0155 , calcd for $\mathrm{C}_{20} \mathrm{H}_{17}{ }^{79} \mathrm{BrN}_{2} \mathrm{O}_{2} \mathrm{~S}$ 428.0194, found 428.0180;

Anal. calcd for $\mathrm{C}_{20} \mathrm{H}_{17} \mathrm{BrN}_{2} \mathrm{O}_{2} \mathrm{~S}: \mathrm{C}, 55.95 ; \mathrm{H}, 3.99 ; \mathrm{N}, 6.52$. found: $\mathrm{C}, 55.98 ; \mathrm{H}, 4.02 ; \mathrm{N}$, 6.52 . 


\section{3-Nitrobenzophenone 4-Methylphenylsulfonylhydrazone (3l)}<smiles>NN=C(c1ccccc1)c1cccc([N+](=O)[O-])c1</smiles>

According to the general procedure, a mixture of 3-nitrobenzophenone $(0.50 \mathrm{~g}, 2.2$ mmol), acetyl chloride ( $0.87 \mathrm{~g}, 11.0 \mathrm{mmol})$, and 4-methylphenylsulfonylhydrazide ( $0.82 \mathrm{~g}$, $4.4 \mathrm{mmol})$ in ethanol $(12 \mathrm{~mL})$ was heated under reflux for $1 \mathrm{~h}$. The crude material was purified by silica gel column chromatography [hexane-ethyl acetate $(4: 1)]$ to afford $3 \mathbf{l}(0.84$ $\mathrm{g}, 97 \%$ ) as an inseparable mixture of two isomers. The ratio of two isomers was determined by HPLC as $2: 1 \sim 3: 1$.

IR $v\left(\right.$ film, $\left.\mathrm{cm}^{-1}\right) 3200,1529,1348,1167,854,814,775,733,691,665$;

${ }^{1} \mathrm{H}-\mathrm{NMR} /{ }^{13} \mathrm{C}-\mathrm{NMR}$ : only a ${ }^{1} \mathrm{H}-\mathrm{NMR}$ spectrum of $E$-/Z-mixture was obtained;

MS m/z 395 (M , 31.0), 240 (100), 211 (89.9), 194 (98.5);

HRMS calcd for $\mathrm{C}_{20} \mathrm{H}_{17} \mathrm{~N}_{3} \mathrm{O}_{4} \mathrm{~S} 395.0940$, found 395.0924;

Anal. calcd for $\mathrm{C}_{20} \mathrm{H}_{17} \mathrm{~N}_{3} \mathrm{O}_{4} \mathrm{~S}: \mathrm{C}, 60.75 ; \mathrm{H}, 4.33 ; \mathrm{N}, 10.63$. found: $\mathrm{C}, 60.61 ; \mathrm{H}, 4.35 ; \mathrm{N}$, 10.57 .

\section{4-Nitrobenzophenone 4-Methylphenylsulfonylhydrazone (3m)}<smiles>[NH3+]N=C(c1ccccc1)c1ccc([N+](=O)[O-])cc1</smiles>

According to the general procedure, a mixture of 4-nitrobenzophenone $(0.31 \mathrm{~g}, 1.3$ mmol), acetyl chloride $(0.21 \mathrm{~g}, 2.7 \mathrm{mmol})$, and 4-methylphenylsulfonylhydrazide $(0.50 \mathrm{~g}$, $2.7 \mathrm{mmol})$ in ethanol $(9 \mathrm{~mL})$ was heated under reflux for $22 \mathrm{~h}$. The crude material was purified by silica gel column chromatography [hexane-ethyl acetate (4:1)] to afford $3 \mathbf{m}-E$ $(0.41 \mathrm{~g}, 78 \%)$ and $3 \mathrm{~m}-Z(83.0 \mathrm{mg}, 16 \%)$ as a yellow solid, respectively. Geometries of both isomers were confirmed by NOESY. 
$E$-isomer: $\mathrm{mp} 139-140^{\circ} \mathrm{C}$ (yellow plates from hexane-ethyl acetate, lit. ${ }^{4)} \mathrm{mp} 130-138^{\circ} \mathrm{C}$ );

IR $v$ (film, $\mathrm{cm}^{-1}$ ) 3202, 1520, 1344, 1167, 862, 777, 737, 700, 681, 656;

${ }^{1} \mathrm{H}-\mathrm{NMR}\left(600 \mathrm{MHz}, \mathrm{C}_{6} \mathrm{D}_{6}\right) \delta 1.87(3 \mathrm{H}, \mathrm{s}), 6.57(2 \mathrm{H}, \mathrm{d}, J=7.2 \mathrm{~Hz}), 6.83(2 \mathrm{H}, \mathrm{d}, J=7.8$ $\mathrm{Hz}), 6.94-6.98(3 \mathrm{H}, \mathrm{m}), 7.24(2 \mathrm{H}, \mathrm{d}, J=9.0 \mathrm{~Hz}), 7.69(2 \mathrm{H}, \mathrm{d}, J=9.0 \mathrm{~Hz}), 7.77(1 \mathrm{H}, \mathrm{s})$, $7.90(2 \mathrm{H}, \mathrm{d}, J=7.8 \mathrm{~Hz})$;

${ }^{13} \mathrm{C}-\mathrm{NMR}\left(100 \mathrm{MHz}, \mathrm{CDCl}_{3}\right) \delta 21.7,123.4,127.8,128.1,129.73,129.75,129.8,130.1$, 130.6, 135.1, 142.1, 144.5, 148.2, 151.2;

MS m/z 395 (M+, 29.7), 240 (82.8), 211 (99.5), 194 (100);

HRMS calcd for $\mathrm{C}_{20} \mathrm{H}_{17} \mathrm{~N}_{3} \mathrm{O}_{4} \mathrm{~S} 395.0940$, found 395.0953.

Z-isomer: $\mathrm{mp} 183-185^{\circ} \mathrm{C}$ (light yellow plates from hexane-ethyl acetate);

IR $v\left(\right.$ film, $\left.\mathrm{cm}^{-1}\right)$ 3190, 1520, 1348, 1165, 849, 758, 700, 671;

${ }^{1} \mathrm{H}-\mathrm{NMR}\left(600 \mathrm{MHz}, \mathrm{C}_{6} \mathrm{D}_{6}\right) \delta 1.84(3 \mathrm{H}, \mathrm{s}), 6.28(2 \mathrm{H}, \mathrm{d}, J=8.7 \mathrm{~Hz}), 6.79(2 \mathrm{H}, \mathrm{d}, J=8.1$ $\mathrm{Hz}), 7.03-7.04(3 \mathrm{H}, \mathrm{m}), 7.34-7.36(2 \mathrm{H}, \mathrm{m}), 7.53(2 \mathrm{H}, \mathrm{d}, J=8.7 \mathrm{~Hz}), 7.59(1 \mathrm{H}, \mathrm{s}), 8.02(2 \mathrm{H}$, d, $J=8.1 \mathrm{~Hz})$;

${ }^{13} \mathrm{C}-\mathrm{NMR}\left(100 \mathrm{MHz}, \mathrm{CDCl}_{3}\right) \delta 21.7,124.8,127.3,127.8,128.4,129.68,129.72,130.3$, 135.0, 135.4, 137.8, 144.4, 148.5, 151.8;

MS m/z $395\left(\mathrm{M}^{+}, 18.7\right), 240$ (77.8), 211 (90.5), 194 (100);

HRMS calcd for $\mathrm{C}_{20} \mathrm{H}_{17} \mathrm{~N}_{3} \mathrm{O}_{4} \mathrm{~S} 395.0940$, found 395.0942.

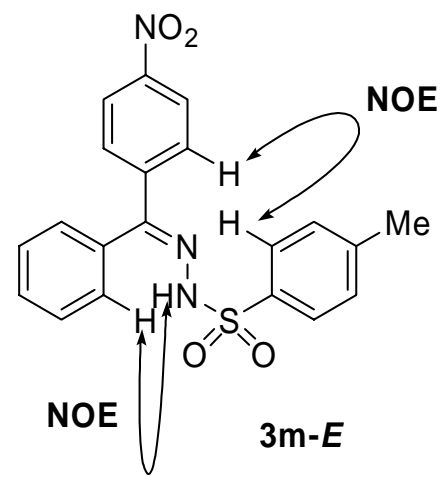<smiles>[CH]Cc1cc(C)ccc1S(=O)(=O)N/N=C(\c1ccccc1)c1ccc([N+](=O)[O-])c(CCN(CC)OCC)c1</smiles> 


\section{4-Methoxybenzophenone 4-Methylphenylsulfonylhydrazone (3n)}<smiles>COc1ccc(/C(=N/NSC)c2ccccc2)cc1</smiles>

According to the general procedure, a mixture of 4-methoxybenzophenone $(0.30 \mathrm{~g}, 1.4$ mmol), acetyl chloride $(0.22 \mathrm{~g}, 2.8 \mathrm{mmol})$, and 4-methylphenylsulfonylhydrazide $(0.79 \mathrm{~g}$, $4.2 \mathrm{mmol})$ in ethanol $(15 \mathrm{~mL})$ was heated under reflux for $43 \mathrm{~h}$. The crude material was purified by silica gel column chromatography [hexane-ethyl acetate (4:1)] to afford $3 \mathbf{n}$ as an inseparable mixture of two isomers $(0.12 \mathrm{~g}, 22 \%)$. The ratio of two isomers was determined by ${ }^{1} \mathrm{H}-\mathrm{NMR}$ spectrum as $E: Z=1: 1$. Recrystallization gave the single isomer ( $E$-isomer). Geometries of both isomers were confirmed by NOESY.

IR $v\left(\right.$ film, $\left.\mathrm{cm}^{-1}\right)$ 3200, 1161, 837, 775, 700, 664;

$\mathrm{MS} m / z 380\left(\mathrm{M}^{+}, 18.2\right), 225$ (100);

HRMS calcd for $\mathrm{C}_{21} \mathrm{H}_{20} \mathrm{~N}_{2} \mathrm{O}_{3} \mathrm{~S} 380.1195$, found 380.1196;

Anal. calcd for $\mathrm{C}_{21} \mathrm{H}_{20} \mathrm{~N}_{2} \mathrm{O}_{3} \mathrm{~S}$ : C, 66.29; H, 5.30; N, 7.36. found: C, 66.29; H, 5.30; N, 7.41.

E-isomer: ${ }^{1} \mathrm{H}-\mathrm{NMR}\left(400 \mathrm{MHz}, \mathrm{CDCl}_{3}\right) \delta 2.42(3 \mathrm{H}, \mathrm{s}), 3.78(3 \mathrm{H}, \mathrm{s}), 6.79(2 \mathrm{H}, \mathrm{d}, J=9.0$ $\mathrm{Hz}), 7.09$ (2H, dd, $J=6.4,3.2 \mathrm{~Hz}), 7.32(2 \mathrm{H}, \mathrm{d}, J=8.0 \mathrm{~Hz}), 7.37(2 \mathrm{H}, \mathrm{d}, J=9.0 \mathrm{~Hz}), 7.42$ $(1 \mathrm{H}, \mathrm{s}), 7.49-7.50(3 \mathrm{H}, \mathrm{m}), 7.84(2 \mathrm{H}, \mathrm{d}, J=8.0 \mathrm{~Hz})$;

${ }^{13} \mathrm{C}-\mathrm{NMR}\left(100 \mathrm{MHz}, \mathrm{CDCl}_{3}\right) \delta 21.6,55.3,113.5,127.8,128.1,129.01,129.04,129.5$, $129.6,129.9,131.2,135.5,143.9,154.1,160.9$.<smiles>COc1ccc(C2=NNC(C)c3ccccc3C2c2ccccc2)c([N+](=O)[O-])c1</smiles><smiles>CCOCCN(S)N=C(c1ccccc1)c1ccc(OC)cc1</smiles>

$3 n-Z$ 


\section{2-Bromobenzophenone 4-Methylphenylsulfonylhydrazone (3o)}<smiles>NN=C(c1ccccc1)c1ccccc1Br</smiles>

A mixture of 2-bromobenzoic acid $(1.0 \mathrm{~g}, 5.0 \mathrm{mmol})$ and thionyl chloride $(1.2 \mathrm{~g}, 10.0$ $\mathrm{mmol})$ in chloroform $(10 \mathrm{~mL})$ was heated under reflux for $3 \mathrm{~h}$. After the excess thionyl chloride and chloroform had been evaporated, the crude benzoyl chloride was dissolved in benzene $(4 \mathrm{~mL})$ and added to a suspension of aluminum chloride $(0.75 \mathrm{~g}, 5.5 \mathrm{mmol})$ in benzene $(8 \mathrm{~mL})$. After being heated under reflux for $6 \mathrm{~h}$, the reaction mixture was quenched with diluted $\mathrm{HCl}(2 \mathrm{~mL})$. The mixture was extracted with ethyl acetate $(20 \mathrm{~mL} \times 3)$ and the combined organic layer was washed with brine $(5 \mathrm{~mL})$, and dried over $\mathrm{MgSO}_{4}$. The solvent was evaporated and the residue was purified by silica gel column chromatography [hexane-ethyl acetate (9:1)] to give 2-bromobenzophenone (1.0 g, 77\%) as a colorless oil.

IR $v$ (neat, $\left.\mathrm{cm}^{-1}\right) 1670$;

${ }^{1} \mathrm{H}-\mathrm{NMR}\left(400 \mathrm{MHz}, \mathrm{CDCl}_{3}\right) \delta$ 7.32-7.44 (3H, m), $7.48(2 \mathrm{H}, \mathrm{t}, J=7.5 \mathrm{~Hz}), 7.59(1 \mathrm{H}, \mathrm{d}, J=$ $8.2 \mathrm{~Hz}), 7.63-7.65(1 \mathrm{H}, \mathrm{m}), 7.83(2 \mathrm{H}, \mathrm{d}, J=7.5 \mathrm{~Hz})$;

${ }^{13} \mathrm{C}-\mathrm{NMR}\left(100 \mathrm{MHz}, \mathrm{CDCl}_{3}\right) \delta 119.3,127.0,128.5,128.8,130.0,131.0,133.0,133.5$, 135.9, 140.4, 195.5;

MS $m / z 262\left(\mathrm{M}^{+}+2,32.0\right), 260\left(\mathrm{M}^{+}, 31.4\right), 105(100)$;

HRMS calcd for $\mathrm{C}_{13} \mathrm{H}_{9}{ }^{79} \mathrm{BrO} 259.9837$, found 259.9811 .

According to the general procedure, a mixture of 2-bromobenzophenone (1.5 g, 5.7 mmol), acetyl chloride (2.3 g, $28.7 \mathrm{mmol})$, and 4-methylphenylsulfonylhydrazide ( $2.1 \mathrm{~g}$, $11.5 \mathrm{mmol})$ in ethanol $(25 \mathrm{~mL})$ was heated under reflux for $43 \mathrm{~h}$. The crude material was purified by silica gel column chromatography [hexane-ethyl acetate (4:1)] to afford 30 (1.8 $\mathrm{g}, 74 \%$, single isomer) as a colorless solid.

$\mathrm{mp} 125-126^{\circ} \mathrm{C}$ (colorless prisms from hexane-chloroform, lit. ${ }^{3)} \mathrm{mp} 126^{\circ} \mathrm{C}$ ); IR $v$ (Nujol, $\left.\mathrm{cm}^{-1}\right)$ 3196, 1389, 1167;

${ }^{1} \mathrm{H}-\mathrm{NMR}\left(400 \mathrm{MHz} \mathrm{CDCl}_{3}\right) \delta 2.41(3 \mathrm{H}, \mathrm{s}), 7.10(1 \mathrm{H}, \mathrm{d}, J=7.8 \mathrm{~Hz}), 7.20-7.49(10 \mathrm{H}, \mathrm{m})$, 
$7.70(1 \mathrm{H}, \mathrm{d}, J=7.8 \mathrm{~Hz}), 7.89(2 \mathrm{H}, \mathrm{d}, J=8.3 \mathrm{~Hz})$;

${ }^{13} \mathrm{C}-\mathrm{NMR}\left(100 \mathrm{MHz}, \mathrm{CDCl}_{3}\right) \delta 21.7,121.4,126.9,128.0,128.3,128.6,129.4,129.9,130.0$, 131.5, 132.5, 133.6, 135.0, 135.2, 144.0, 152.0;

MS $m / z 430\left(\mathrm{M}^{+}+2,9.8\right), 428\left(\mathrm{M}^{+}, 9.1\right), 193(100)$;

HRMS calcd for $\mathrm{C}_{20} \mathrm{H}_{17}{ }^{79} \mathrm{BrN}_{2} \mathrm{O}_{2} \mathrm{~S} 428.0194$, found 428.0182;

Anal. calcd for $\mathrm{C}_{20} \mathrm{H}_{17} \mathrm{BrN}_{2} \mathrm{O}_{2} \mathrm{~S}$ : C, 55.95; H, 3.99; N, 6.52. found: C, 55.83; H, 3.87; N, 6.59 .

\section{Results of Screenings}

\section{3-1. Effect of Pd and Cu}

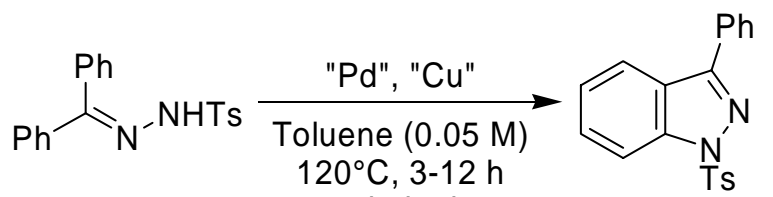
sealed tube

\begin{tabular}{|c|c|c|c|}
\hline Entry & "Pd" (mol\%) & “Cu” (mol\%) & Yield $(\%)^{a, b}$ \\
\hline 1 & --- & --- & $0(99)$ \\
\hline 2 & $\mathrm{Pd}(\mathrm{OAc})_{2}(100)$ & --- & 18 \\
\hline 3 & --- & $\mathrm{Cu}(\mathrm{OAc})_{2}(100)$ & 0 \\
\hline 4 & $\mathrm{Pd}(\mathrm{OAc})_{2}(100)$ & $\mathrm{Cu}(\mathrm{OAc})_{2}(100)$ & 31 \\
\hline 5 & $\operatorname{Pd}(\mathrm{OAc})_{2}(50)$ & $\mathrm{Cu}(\mathrm{OAc})_{2}(100)$ & $18(16)$ \\
\hline 6 & $\mathrm{Pd}(\mathrm{OAc})_{2}(10)$ & $\mathrm{Cu}(\mathrm{OAc})_{2}(100)$ & 5 \\
\hline 7 & $\mathrm{Pd}(\mathrm{OAc})_{2}(10)$ & $\mathrm{CuCl}_{2}(100)$ & trace \\
\hline 8 & $\mathrm{Pd}(\mathrm{OAc})_{2}(10)$ & $\mathrm{CuBr}_{2}(100)$ & trace \\
\hline 9 & $\mathrm{Pd}(\mathrm{OAc})_{2}(50)$ & (Benzoquinone) (100) & $11(19)$ \\
\hline 10 & $\mathrm{PdCl}_{2}(50)$ & $\mathrm{Cu}(\mathrm{OAc})_{2}(100)$ & 0 \\
\hline 11 & $\mathrm{Pd}(\mathrm{OAc})_{2}\left[(o-\mathrm{Tol})_{3} \mathrm{P}\right]_{2}(50)$ & $\mathrm{Cu}(\mathrm{OAc})_{2}(100)$ & trace \\
\hline 12 & $\mathrm{PdCl}_{2}(\mathrm{dppf})(50)$ & $\mathrm{Cu}(\mathrm{OAc})_{2}(100)$ & trace \\
\hline 13 & $\mathrm{PdCl}_{2}(\mathrm{dppp})(50)$ & $\mathrm{Cu}(\mathrm{OAc})_{2}(100)$ & 0 \\
\hline 14 & $\mathrm{PdCl}_{2}(\mathrm{dppb})(50)$ & $\mathrm{Cu}(\mathrm{OAc})_{2}(100)$ & 0 \\
\hline 15 & $\mathrm{Pd}_{2}(\mathrm{dba})_{3}(25)$ & $\mathrm{Cu}(\mathrm{OAc})_{2}(100)$ & 0 \\
\hline 16 & $\mathrm{PdCl}_{2}(\mathrm{COD})_{2}(50)$ & $\mathrm{Cu}(\mathrm{OAc})_{2}(100)$ & $5(47)$ \\
\hline 17 & $\mathrm{PdCl}_{2}(\mathrm{MeCN})_{2}(50)$ & $\mathrm{Cu}(\mathrm{OAc})_{2}(100)$ & 0 \\
\hline
\end{tabular}


${ }^{a}$ Isolated yield. ${ }^{b}$ Recovery yield of starting material in parentheses.

\section{3-2. Effect of Solvents}

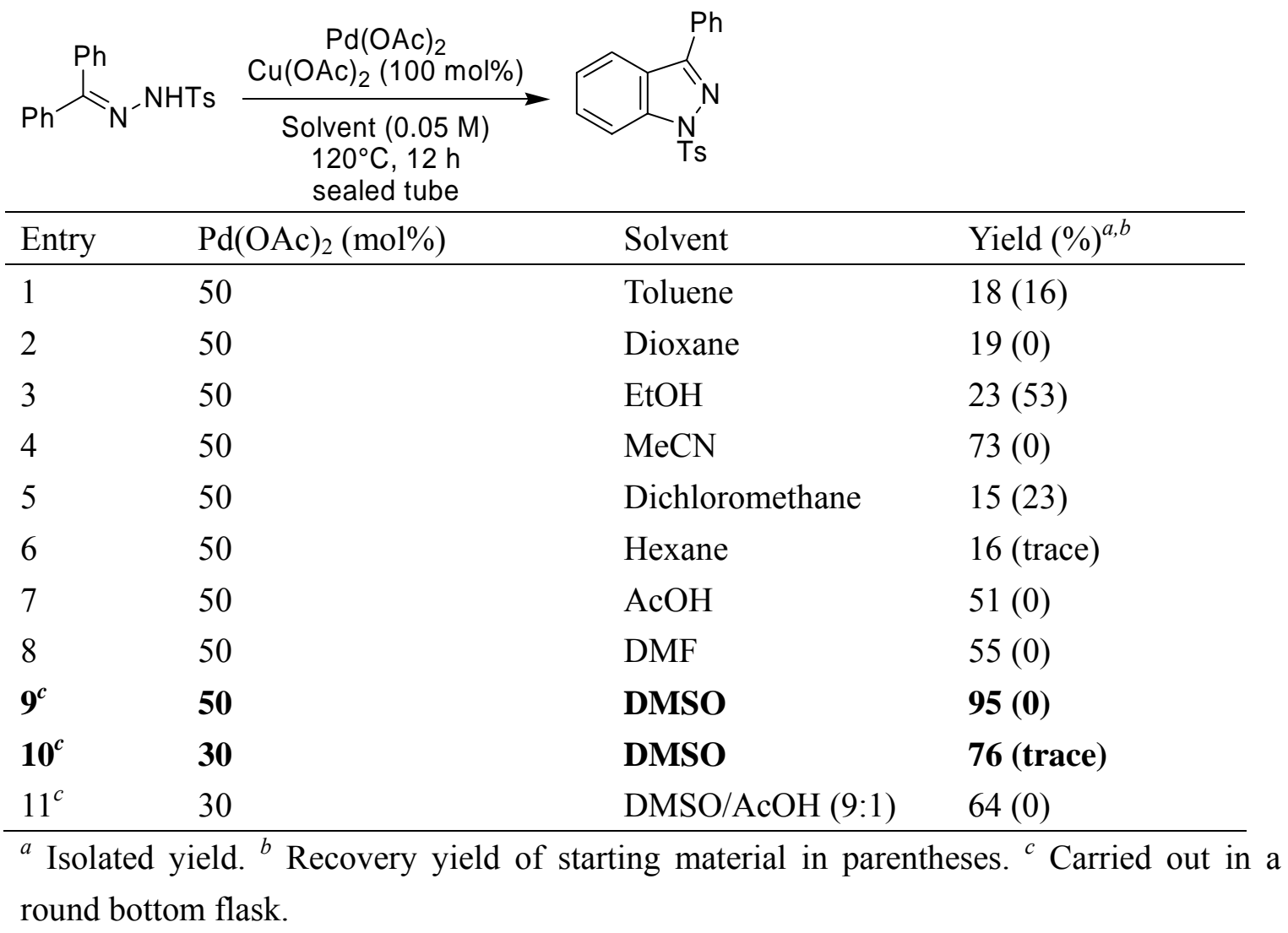

\section{3-3. Effect of Conditions (Temp./Time)}

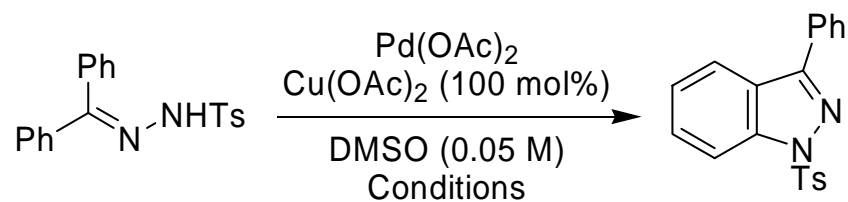

\begin{tabular}{|c|c|c|c|}
\hline Entry & $\mathrm{Pd}(\mathrm{OAc})_{2}(\mathrm{~mol} \%)$ & Conditions & Yield $(\%)^{a, b}$ \\
\hline 1 & 30 & $120^{\circ} \mathrm{C}, 12 \mathrm{~h}$ & 76 (trace) \\
\hline 2 & 30 & $100^{\circ} \mathrm{C}, 12 \mathrm{~h}$ & $64(12)$ \\
\hline 3 & 30 & $80^{\circ} \mathrm{C}, 12 \mathrm{~h}$ & $76(7)$ \\
\hline 4 & 30 & $50^{\circ} \mathrm{C}, 12 \mathrm{~h}$ & $85(0)$ \\
\hline 5 & 30 & $\mathrm{rt}, 12 \mathrm{~h}$ & $5(70)$ \\
\hline
\end{tabular}




\begin{tabular}{lccc}
$\mathbf{6}$ & $\mathbf{2 0}$ & $\mathbf{5 0 ^ { \circ } \mathbf { C } , \mathbf { 1 2 }} \mathbf{~ h}$ & $\mathbf{5 8 ( 3 0 )}$ \\
7 & 15 & $50^{\circ} \mathrm{C}, 36 \mathrm{~h}$ & $48(39)$ \\
8 & 10 & $50^{\circ} \mathrm{C}, 12 \mathrm{~h}$ & $14(61)$ \\
$\mathbf{9}$ & $\mathbf{1 0}$ & $\mathbf{5 0}^{\circ} \mathbf{C}, \mathbf{2 5} \mathbf{~ h}$ & $\mathbf{5 5 ( 4 1 )}$ \\
10 & 10 & $50^{\circ} \mathrm{C}, 62 \mathrm{~h}$ & $44(32)$ \\
\hline${ }^{a}$ Isolated yield. ${ }^{b}$ Recovery yield of starting material in parentheses.
\end{tabular}

\section{3-4. Effect of Oxidants}

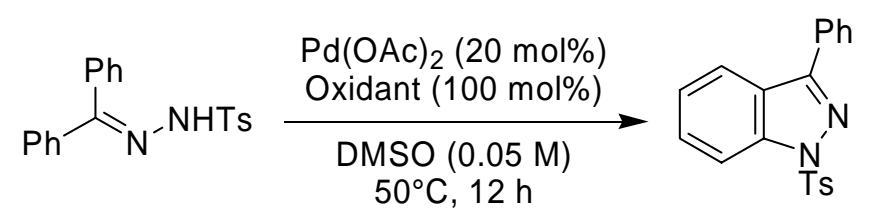

\begin{tabular}{|c|c|c|}
\hline Entry & Oxidant & Yield $(\%)^{a, b}$ \\
\hline 1 & $\mathrm{Cu}(\mathrm{OAc})_{2}$ & $58(30)$ \\
\hline 2 & $\mathrm{Cu}(\mathrm{OAc})_{2}{ }^{c}$ & $58(32)$ \\
\hline 3 & $\mathrm{Cu}(\mathrm{OTs})_{2}$ & $7(66)$ \\
\hline 4 & $\mathrm{Cu}(\mathrm{OMs})_{2}$ & $8(64)$ \\
\hline 5 & $\mathrm{Cu}(\mathrm{OTf})_{2}$ & $13(60)$ \\
\hline 6 & $\mathrm{Cu}\left(\mathrm{OCOCF}_{3}\right)_{2}-\mathrm{H}_{2} \mathrm{O}$ & $30(50)$ \\
\hline 7 & $\mathrm{CuSt}_{2}{ }^{d}$ & $39(53)$ \\
\hline
\end{tabular}

\section{3-5. Effect of Concentration}

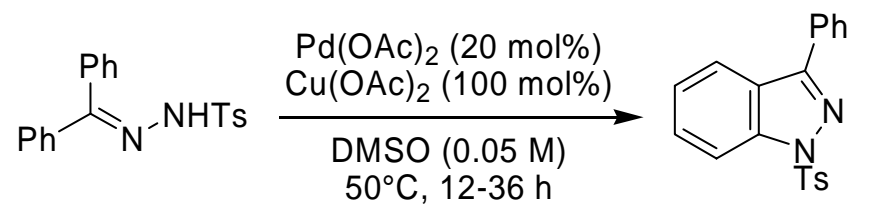

\begin{tabular}{lll}
\hline Entry & Concentration & Yield $(\%)^{a, b}$ \\
\hline 1 & 0.025 & $55(35)$ \\
2 & 0.01 & $50(39)$ \\
3 & 0.05 & $55(41)$ \\
4 & 0.1 & $14(66)$
\end{tabular}




\begin{tabular}{llc}
5 & 0.2 & $13(50)$ \\
6 & 0.3 & $17(34)$ \\
\hline${ }^{a}$ Isolated yield. ${ }^{b}$ Recovery yield of starting material in parentheses.
\end{tabular}

\section{3-6. Effect of Additives}

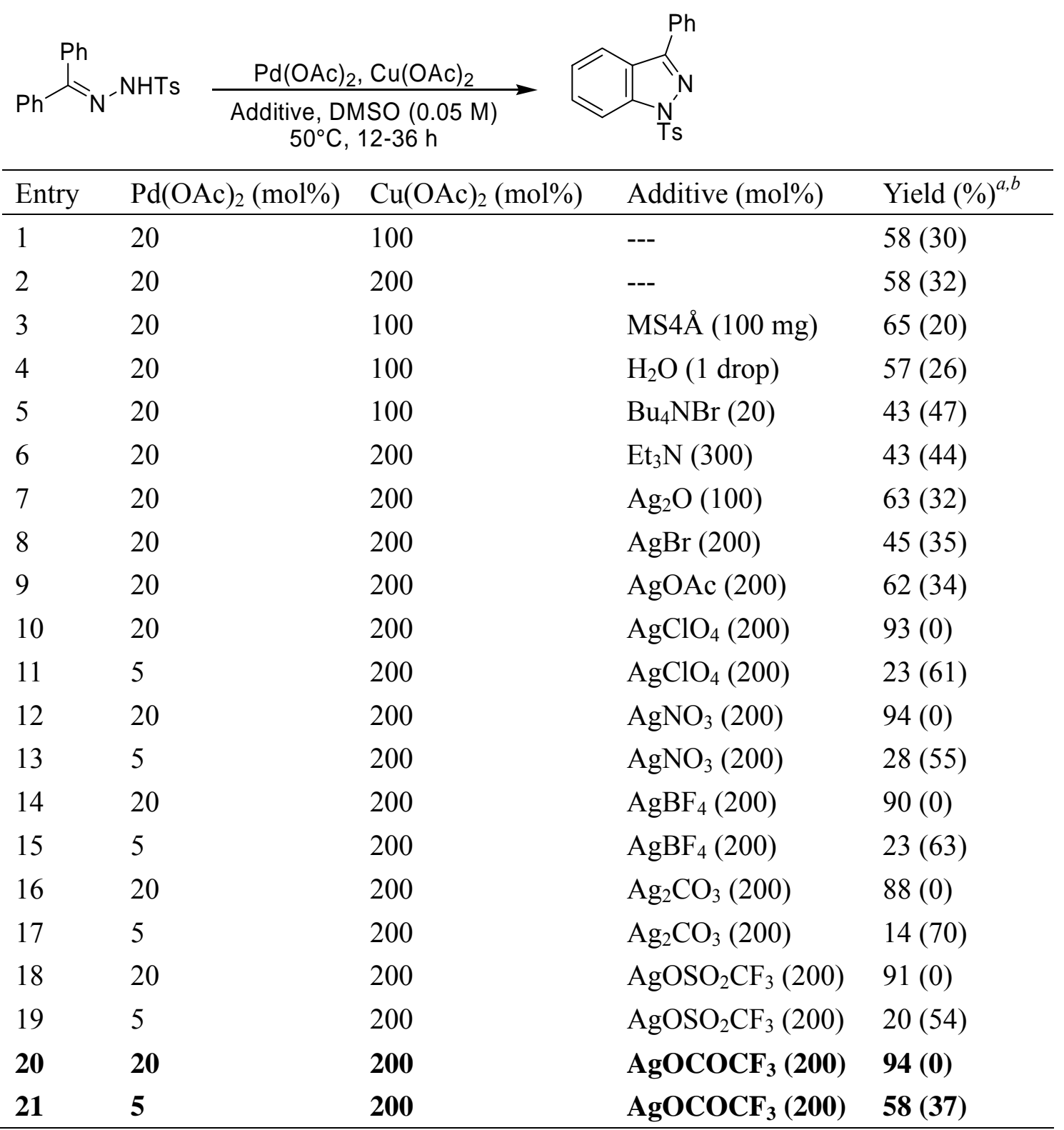

${ }^{a}$ Isolated yield. ${ }^{b}$ Recovery yield of starting material in parentheses. 
3-7. Optimization of $\mathrm{Pd}(\mathrm{OAc})_{2} / \mathrm{Cu}(\mathrm{OAc})_{2} / \mathrm{AgOCOCF}_{3}$ System

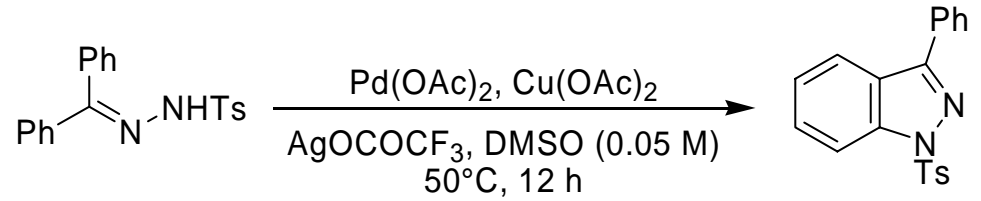

\begin{tabular}{lll}
\hline Entry & $\mathrm{Pd}(\mathrm{OAc})_{2} / \mathrm{Cu}(\mathrm{OAc})_{2} / \mathrm{AgOCOCF}_{3}\left(\mathrm{~mol}^{2}\right)$ & Yield $(\%)^{a, b}$ \\
\hline 1 & $20 / 0 / 0$ & $16(74)$ \\
$2^{c}$ & $0 / 100 / 0$ & $\mathrm{NR}$ \\
3 & $0 / 0 / 200$ & $\mathrm{NR}$ \\
4 & $20 / 200 / 0$ & $58(32)$ \\
5 & $20 / 0 / 200$ & $26(68)$ \\
6 & $0 / 200 / 200$ & $\mathrm{NR}$ \\
7 & $20 / 200 / 200$ & $94(0)$ \\
8 & $10 / 200 / 200$ & $87(0)$ \\
9 & $5 / 200 / 200$ & $58(37)$ \\
10 & $10 / 100 / 100$ & $33(59)$ \\
$\mathbf{1 1}$ & $\mathbf{1 0} / \mathbf{1 0 0} / 200$ & $\mathbf{9 0}(\mathbf{0})$ \\
12 & $20^{c} / 200 / 0$ & $63(20)$ \\
\hline
\end{tabular}

${ }^{a}$ Isolated yield. ${ }^{b}$ Recovery yield of starting material in parentheses. ${ }^{c} \mathrm{Pd}\left(\mathrm{OCOCF}_{3}\right)_{2}$ was used instead of $\mathrm{Pd}(\mathrm{OAc})_{2}$.

1-(4-Methylphenylsulfonyl)-3-phenylindazole (2) (Table 1, Entry 11)<smiles>[As]n1nc(-c2ccccc2)c2ccccc21</smiles>

A mixture of 1 (50.0 mg, $0.14 \mathrm{mmol}), \mathrm{Pd}(\mathrm{OAc})_{2}$ (3.2 mg, $\left.0.014 \mathrm{mmol}\right), \mathrm{Cu}(\mathrm{OAc})_{2}(25.9$ $\mathrm{mg}, 0.14 \mathrm{mmol})$, and $\mathrm{AgOCOCF}_{3}(63.0 \mathrm{mg}, 0.29 \mathrm{mmol})$ in DMSO $(2.9 \mathrm{~mL})$ was stirred at $50^{\circ} \mathrm{C}$ for $12 \mathrm{~h}$. The reaction mixture was extracted with ethyl acetate $(5 \mathrm{~mL} \times 3)$ and the combined organic layer was washed with brine $(10 \mathrm{~mL})$, and dried over $\mathrm{MgSO}_{4}$. The solvent was evaporated and the residue was purified by silica gel column chromatography [hexane-ethyl acetate (9:1)] to afford $2(44.7 \mathrm{mg}, 90 \%)$ as a colorless solid. 
$\mathrm{mp} 115-116^{\circ} \mathrm{C}$ (colorless scales from hexane-ethyl acetate, lit. ${ }^{5)} \mathrm{mp} 115-116^{\circ} \mathrm{C}$ );

IR $\vee\left(\right.$ Nujol, $\left.\mathrm{cm}^{-1}\right)$ 1366, 1174;

${ }^{1} \mathrm{H}-\mathrm{NMR}\left(400 \mathrm{MHz}, \mathrm{CDCl}_{3}\right) \delta 2.35(3 \mathrm{H}, \mathrm{s}), 7.15-7.53(7 \mathrm{H}, \mathrm{m}), 7.90-7.94(5 \mathrm{H}, \mathrm{m}), 8.26$ $(1 \mathrm{H}, \mathrm{d}, J=8.5 \mathrm{~Hz})$;

${ }^{13} \mathrm{C}-\mathrm{NMR}\left(100 \mathrm{MHz}, \mathrm{CDCl}_{3}\right)$ 21.5, 113.5, 121.6, 124.3, 124.4, 127.5, 128.2, 128.8, 129.0, $129.5,129.7,131.4,134.6,141.8,145.2,151.6$.

MS $m / z 348\left(\mathrm{M}^{+}, 49.3\right), 193(100)$;

HRMS calcd for $\mathrm{C}_{20} \mathrm{H}_{16} \mathrm{~N}_{2} \mathrm{O}_{2} \mathrm{~S} 348.0932$, found 348.0887.

4. General Procedure for Pd-Catalyzed C-H Amination of Hydrazones (Tables 2 and 3)

A mixture of hydrazone 3 (1.0 eq.), $\mathrm{Pd}(\mathrm{OAc})_{2}(10 \mathrm{~mol} \%), \mathrm{Cu}(\mathrm{OAc})_{2}(100 \mathrm{~mol} \%)$, and $\mathrm{AgOCOCF}_{3}(200 \mathrm{~mol} \%)$ in DMSO $(0.05 \mathrm{M})$ was stirred at $50^{\circ} \mathrm{C}$. The reaction mixture was extracted with ethyl acetate $(5 \mathrm{~mL}$ x 3$)$ and the combined organic layer was washed with brine $(10 \mathrm{~mL})$, and dried over $\mathrm{MgSO}_{4}$. The solvent was evaporated and the residue was purified by silica gel column chromatography.

6-Methoxy-3-(4-methoxyphenyl)-1-(4-methylphenylsulfonyl)indazole (4a) (Table 2, Entry 1)<smiles>COc1ccc(-c2nn(S)c3cc(OC)ccc23)cc1</smiles>

According to the general procedure, a mixture of $3 \mathbf{a}(50.0 \mathrm{mg}, 0.12 \mathrm{mmol}), \mathrm{Pd}(\mathrm{OAc})_{2}$ (2.7 mg, $0.012 \mathrm{mmol}), \mathrm{Cu}(\mathrm{OAc})_{2}(22.1 \mathrm{mg}, 0.12 \mathrm{mmol})$, and $\mathrm{AgOCOCF}_{3}(53.8 \mathrm{mg}, 0.24$ $\mathrm{mmol})$ in DMSO $(2.4 \mathrm{~mL})$ was stirred for $24 \mathrm{~h}$. The crude product was purified by silica gel column chromatography [hexane-ethyl acetate $(3: 1)$ ] to afford $\mathbf{4 a}(4.6 \mathrm{mg}, 9 \%)$ and $\mathbf{3 a}$ (15.4 mg, 31\%).

$\mathrm{mp} 160-161^{\circ} \mathrm{C}$ (colorless prisms from hexane-ethyl acetate); 
IR $v\left(\right.$ film, $\left.\mathrm{cm}^{-1}\right) 1611,1175,669$;

${ }^{1} \mathrm{H}-\mathrm{NMR}\left(400 \mathrm{MHz}, \mathrm{CDCl}_{3}\right) \delta 2.35(3 \mathrm{H}, \mathrm{s}), 3.86(3 \mathrm{H}, \mathrm{s}), 3.96(3 \mathrm{H}, \mathrm{s}), 6.96(1 \mathrm{H}, \mathrm{dd}, J=8.6$, $2.2 \mathrm{~Hz}), 7.00(2 \mathrm{H}, \mathrm{d}, J=8.8 \mathrm{~Hz}), 7.22(2 \mathrm{H}, \mathrm{d}, J=8.0 \mathrm{~Hz}), 7.68(1 \mathrm{H}, \mathrm{d}, J=2.2 \mathrm{~Hz}), 7.73$ $(1 \mathrm{H}, \mathrm{d}, J=8.6 \mathrm{~Hz}), 7.82(2 \mathrm{H}, \mathrm{d}, J=8.8 \mathrm{~Hz}), 7.87(2 \mathrm{H}, \mathrm{d}, J=8.0 \mathrm{~Hz})$;

${ }^{13} \mathrm{C}-\mathrm{NMR}\left(100 \mathrm{MHz}, \mathrm{CDCl}_{3}\right) \delta 21.7,55.4,55.9,95.7,114.1,115.3,118.5,122.3,124.0$, $127.5,129.4,129.6,134.7,143.6,145.0,151.4,160.6,161.0$;

MS $m / z 408\left(\mathrm{M}^{+}, 46.3\right), 253$ (100), 225 (33.6);

HRMS calcd for $\mathrm{C}_{22} \mathrm{H}_{20} \mathrm{~N}_{2} \mathrm{O}_{4} \mathrm{~S} 408.1144$, found 408.1129 .

6-Methyl-3-(4-methylphenyl)-1-(4-methylphenylsulfonyl)indazole (4b) (Table 2, Entry 2)<smiles>Cc1ccc(-c2nn(S)c3cc(C)ccc23)cc1</smiles>

According to the general procedure, a mixture of $\mathbf{3 b}(50.0 \mathrm{mg}, 0.13 \mathrm{mmol}), \mathrm{Pd}(\mathrm{OAc})_{2}$ (3.0 mg, $0.013 \mathrm{mmol}), \mathrm{Cu}(\mathrm{OAc})_{2}(24.0 \mathrm{mg}, 0.13 \mathrm{mmol})$, and $\mathrm{AgOCOCF}_{3}(58.4 \mathrm{mg}, 0.26$ $\mathrm{mmol})$ in DMSO $(2.6 \mathrm{~mL})$ was stirred for $24 \mathrm{~h}$. The crude product was purified by silica gel column chromatography [hexane-ethyl acetate (9:1)] to afford $\mathbf{4 b}(26.0 \mathrm{mg}, 52 \%)$ and $\mathbf{3 b}$ (14.5 mg, 29\%).

$\mathrm{mp} 158-159^{\circ} \mathrm{C}$ (colorless prisms from hexane-ethyl acetate);

IR $v$ (film, $\mathrm{cm}^{-1}$ ) 1377, 1175, 679, 667;

${ }^{1} \mathrm{H}-\mathrm{NMR}\left(400 \mathrm{MHz}, \mathrm{CDCl}_{3}\right) \delta 2.34(3 \mathrm{H}, \mathrm{s}), 2.42(3 \mathrm{H}, \mathrm{s}), 2.56(3 \mathrm{H}, \mathrm{s}), 7.18(1 \mathrm{H}, \mathrm{d}, J=8.4$ $\mathrm{Hz}), 7.21(2 \mathrm{H}, \mathrm{d}, J=8.0 \mathrm{~Hz}), 7.29(2 \mathrm{H}, \mathrm{d}, J=8.0 \mathrm{~Hz}), 7.77(1 \mathrm{H}, \mathrm{d}, J=8.4 \mathrm{~Hz}), 7.79(2 \mathrm{H}, \mathrm{d}$, $J=8.0 \mathrm{~Hz}), 7.88(2 \mathrm{H}, \mathrm{d}, J=8.0 \mathrm{~Hz}), 8.04(1 \mathrm{H}, \mathrm{s})$;

${ }^{13} \mathrm{C}-\mathrm{NMR}\left(100 \mathrm{MHz}, \mathrm{CDCl}_{3}\right) \delta 21.4,21.5,22.0,113.3,121.2,122.5,126.2,127.5,128.0$, 128.7, 129.4, 129.7, 134.9, 139.5, 139.7, 142.5, 145.0, 151.6;

MS $m / z 376\left(\mathrm{M}^{+}, 60.8\right), 221$ (100), 193 (37.7);

HRMS calcd for $\mathrm{C}_{22} \mathrm{H}_{20} \mathrm{~N}_{2} \mathrm{O}_{2} \mathrm{~S} 376.1246$, found 376.1225. 
5-Methoxy-3-(3-methoxyphenyl)-1-(4-methylphenylsulfonyl)indazole (4c) (Table 2, Entry 3)<smiles>COc1cccc(-c2nn(S)c3ccc(OC)cc23)c1</smiles>

According to the general procedure, a mixture of $3 c$ (50.0 $\mathrm{mg}, 0.12 \mathrm{mmol}), \mathrm{Pd}(\mathrm{OAc})_{2}$ (2.7 mg, $0.012 \mathrm{mmol}), \mathrm{Cu}(\mathrm{OAc})_{2}\left(22.1 \mathrm{mg}, 0.12 \mathrm{mmol}\right.$ ), and $\mathrm{AgOCOCF}_{3}(53.8 \mathrm{mg}, 0.24$ $\mathrm{mmol})$ in DMSO $(2.4 \mathrm{~mL})$ was stirred for $16 \mathrm{~h}$. The crude product was purified by silica gel column chromatography [hexane-ethyl acetate (4:1)] to afford 4c (48.0 mg, 96\%).

mp $119-120^{\circ} \mathrm{C}$ (colorless plates from hexane-ethyl acetate);

IR $v$ (film, $\left.\mathrm{cm}^{-1}\right)$ 1177, 812, 669;

${ }^{1} \mathrm{H}-\mathrm{NMR}\left(400 \mathrm{MHz}, \mathrm{CDCl}_{3}\right) \delta 2.34(3 \mathrm{H}, \mathrm{s}), 3.85(3 \mathrm{H}, \mathrm{s}), 3.88(3 \mathrm{H}, \mathrm{s}), 7.01(1 \mathrm{H}, \mathrm{dt}, J=6.8$, $2.4 \mathrm{~Hz}), 7.20-7.25(4 \mathrm{H}, \mathrm{m}), 7.40-7.42(3 \mathrm{H}, \mathrm{m}), 7.86(2 \mathrm{H}, \mathrm{d}, J=8.4 \mathrm{~Hz}), 8.14(1 \mathrm{H}, \mathrm{d}, J=$ $8.8 \mathrm{~Hz}$;

${ }^{13} \mathrm{C}-\mathrm{NMR}\left(100 \mathrm{MHz}, \mathrm{CDCl}_{3}\right) \delta 21.5,55.4,55.8,102.0,113.6,114.5,115.1,119.7,120.5$, 125.2, 127.5, 129.7, 129.8, 132.8, 134.5, 137.1, 145.1, 151.4, 157.2, 159.9;

MS $m / z 408\left(\mathrm{M}^{+}, 91.3\right), 253$ (100);

HRMS calcd for $\mathrm{C}_{22} \mathrm{H}_{20} \mathrm{~N}_{2} \mathrm{O}_{4} \mathrm{~S} 408.1144$, found 408.1127 .

5-Methoxy-1-(4-methylphenylsulfonyl)-3-(3-nitrophenyl)indazole (4d) (Table 2, Entry

4)<smiles>COc1ccc2c(c1)c(-c1cccc([N+](=O)[O-])c1)nn2S</smiles>

According to the general procedure, a mixture of $3 \mathbf{d}(50.0 \mathrm{mg}, 0.12 \mathrm{mmol}), \mathrm{Pd}(\mathrm{OAc})_{2}$ (2.6 mg, $0.012 \mathrm{mmol}), \mathrm{Cu}(\mathrm{OAc})_{2}\left(21.3 \mathrm{mg}, 0.12 \mathrm{mmol}\right.$ ), and $\mathrm{AgOCOCF}_{3}$ (51.9 mg, 0.24 $\mathrm{mmol}$ ) in DMSO $(2.4 \mathrm{~mL})$ was stirred for $19 \mathrm{~h}$. The crude product was purified by silica gel column chromatography [hexane-ethyl acetate (4:1)] to afford 4d (49.1 $\mathrm{mg}, 99 \%)$ as a 
colorless solid.

$\mathrm{mp} 194^{\circ} \mathrm{C}$ (colorless needles from hexane-ethyl acetate);

IR $v$ (film, $\left.\mathrm{cm}^{-1}\right)$ 1537, 1379, 1348, 1236, 1177, 729, 671;

${ }^{1} \mathrm{H}-\mathrm{NMR}\left(600 \mathrm{MHz}, \mathrm{CDCl}_{3}\right) \delta 2.36(3 \mathrm{H}, \mathrm{s}), 3.88(3 \mathrm{H}, \mathrm{s}), 7.22(1 \mathrm{H}, \mathrm{d}, J=2.4 \mathrm{~Hz})$, 7.25-7.27 (3H, m), $7.70(1 \mathrm{H}, \mathrm{t}, J=7.8 \mathrm{~Hz}), 7.89(2 \mathrm{H}, \mathrm{d}, J=8.4 \mathrm{~Hz}), 8.18(1 \mathrm{H}, \mathrm{d}, J=9.6$ $\mathrm{Hz}), 8.24(1 \mathrm{H}, \mathrm{dt}, J=7.8,1.2 \mathrm{~Hz}), 8.31(1 \mathrm{H}, \mathrm{ddd}, J=7.8,2.3,1.2 \mathrm{~Hz}), 8.71(1 \mathrm{H}, \mathrm{s})$;

${ }^{13} \mathrm{C}-\mathrm{NMR}\left(150 \mathrm{MHz}, \mathrm{CDCl}_{3}\right) \delta 21.6,55.9,101.1,114.7,120.2,122.8,123.9,124.4,127.6$, $129.9,130.0,133.4,133.8,134.3,137.1,145.5,148.5,148.6,157.5$;

MS $m / z 423\left(\mathrm{M}^{+}, 100\right), 268$ (54.4);

Anal. calcd for $\mathrm{C}_{21} \mathrm{H}_{17} \mathrm{~N}_{3} \mathrm{O}_{5} \mathrm{~S}$ : C, 59.57; H, 4.05; N, 9.92. found: C, 59.37; H, 4.04; N, 9.76.

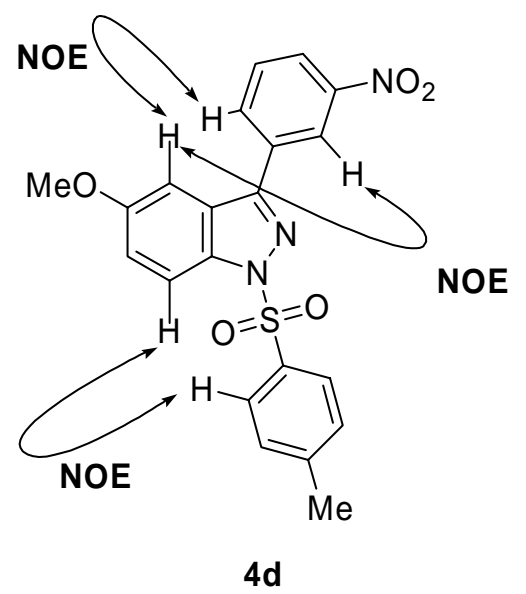

3-(3-Cyanophenyl)-5-methoxy-1-(4-methylphenylsulfonyl)indazole (4e) (Table 2, Entry 5)<smiles>COc1ccc2c(c1)c(-c1cccc(C#N)c1)nn2S</smiles>

According to the general procedure, a mixture of $3 \mathbf{e}(50.0 \mathrm{mg}, 0.12 \mathrm{mmol}), \mathrm{Pd}(\mathrm{OAc})_{2}$ (2.8 mg, $0.012 \mathrm{mmol}), \mathrm{Cu}(\mathrm{OAc})_{2}\left(22.4 \mathrm{mg}, 0.12 \mathrm{mmol}\right.$ ), and $\mathrm{AgOCOCF}_{3}$ (54.5 mg, 0.25 $\mathrm{mmol})$ in DMSO $(2.5 \mathrm{~mL})$ was stirred for $10 \mathrm{~h}$. The crude product was purified by silica gel 
column chromatography [hexane-ethyl acetate $(2: 1)$ ] to afford $4 \mathbf{e}(48.7 \mathrm{mg}, 98 \%)$ as a colorless solid.

$\mathrm{mp} 187-189^{\circ} \mathrm{C}$ (colorless prisms from hexane-ethyl acetate);

IR $v\left(\right.$ film, $\mathrm{cm}^{-1}$ ) 2232, 1491, 1381, 1238, 1190, 1177, 1086, 883, 802, 752, 669;

${ }^{1} \mathrm{H}-\mathrm{NMR}\left(600 \mathrm{MHz}, \mathrm{C}_{6} \mathrm{D}_{6}\right) \delta 1.68(3 \mathrm{H}, \mathrm{s}), 3.24(3 \mathrm{H}, \mathrm{s}), 6.58(2 \mathrm{H}, \mathrm{d}, J=8.1 \mathrm{~Hz}), 6.78(1 \mathrm{H}$, t, $J=7.4 \mathrm{~Hz}), 6.81(1 \mathrm{H}, \mathrm{s}), 7.00(1 \mathrm{H}, \mathrm{d}, J=7.4 \mathrm{~Hz}), 7.03(1 \mathrm{H}, \mathrm{dd}, J=9.0,2.4 \mathrm{~Hz}), 7.70$ $(1 \mathrm{H}, \mathrm{d}, J=7.4 \mathrm{~Hz}), 7.76(1 \mathrm{H}, \mathrm{s}), 7.88(2 \mathrm{H}, \mathrm{d}, J=8.1 \mathrm{~Hz}), 8.28(1 \mathrm{H}, \mathrm{d}, J=9.0 \mathrm{~Hz})$;

${ }^{13} \mathrm{C}-\mathrm{NMR}\left(150 \mathrm{MHz}, \mathrm{C}_{6} \mathrm{D}_{6}\right) \delta 21.0,55.3,101.9,113.9,115.2,118.2,120.1,124.9,128.3$, $129.5,129.8,131.3,131.8,132.3,133.5,135.7,137.9,145.2,148.9,157.9$;

MS $m / z 403\left(\mathrm{M}^{+}, 100\right), 248$ (66.9);

HRMS calcd for $\mathrm{C}_{22} \mathrm{H}_{17} \mathrm{~N}_{3} \mathrm{O}_{3} \mathrm{~S} 403.0991$, found 403.1003 .

Anal. calcd for $\mathrm{C}_{22} \mathrm{H}_{17} \mathrm{~N}_{3} \mathrm{O}_{3} \mathrm{~S}: \mathrm{C}, 65.49 ; \mathrm{H}, 4.25 ; \mathrm{N}, 10.42$. found: C, 65.62; H, 4.30; N, 10.38 .

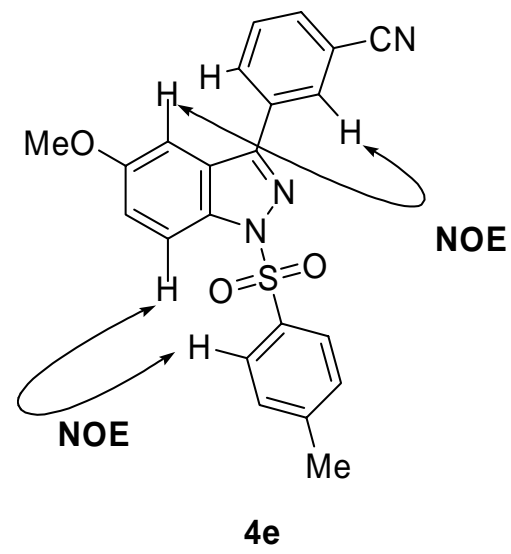

3-(3-Ethoxycarbonylphenyl)-5-methoxy-1-(4-methylphenylsulfonyl)indazole

(4f) (Table 2, Entry 6)<smiles>CCOC(=O)c1cccc(-c2nn(S)c3ccc(OC)cc23)c1</smiles>

According to the general procedure, a mixture of $3 f(50.0 \mathrm{mg}, 0.11 \mathrm{mmol}), \mathrm{Pd}(\mathrm{OAc})_{2}$ 
(2.5 mg, $0.011 \mathrm{mmol}), \mathrm{Cu}(\mathrm{OAc})_{2}(20.1 \mathrm{mg}, 0.11 \mathrm{mmol})$, and $\mathrm{AgOCOCF}_{3}$ (48.8 mg, 0.22 $\mathrm{mmol}$ ) in DMSO $(3.0 \mathrm{~mL})$ was stirred for $24 \mathrm{~h}$. The crude product was purified by silica gel column chromatography [hexane-ethyl acetate $(6: 1)$ ] to afford $\mathbf{4 f}(20.3 \mathrm{mg}, 41 \%)$ and $\mathbf{3 f}$ (25.6 mg, 51\%, A : B = 1: 1).

$\mathrm{mp} 125^{\circ} \mathrm{C}$ (colorless plates from hexane-ethyl acetate);

IR $v\left(\right.$ film cm$\left.~^{-1}\right)$ 2928, 1720, 1381, 1232, 1177, 1078, 669;

${ }^{1} \mathrm{H}-\mathrm{NMR}\left(600 \mathrm{MHz}, \mathrm{C}_{6} \mathrm{D}_{6}\right) \delta 0.97(3 \mathrm{H}, \mathrm{t}, J=7.2 \mathrm{~Hz}), 1.61(3 \mathrm{H}, \mathrm{s}) 3.23(3 \mathrm{H}, \mathrm{s}), 4.09(2 \mathrm{H}, \mathrm{q}$, $J=7.2 \mathrm{~Hz}), 6.50(2 \mathrm{H}, \mathrm{d}, J=8.7 \mathrm{~Hz}), 7.04-7.05(2 \mathrm{H}, \mathrm{m}), 7.10(1 \mathrm{H}, \mathrm{t}, J=8.1 \mathrm{~Hz}), 7.91(2 \mathrm{H}$, $\mathrm{d}, J=8.7 \mathrm{~Hz}), 7.96(1 \mathrm{H}, \mathrm{d}, J=8.1 \mathrm{~Hz}), 8.10(1 \mathrm{H}, \mathrm{d}, J=8.1 \mathrm{~Hz}), 8.31(1 \mathrm{H}, \mathrm{dd}, J=8.7,0.9$ $\mathrm{Hz}), 8.74(1 \mathrm{H}, \mathrm{s})$;

${ }^{13} \mathrm{C}-\mathrm{NMR}\left(600 \mathrm{MHz}, \mathrm{C}_{6} \mathrm{D}_{6}\right), \delta 14.1,21.0,55.0,61.0,101.7,115.1,120.1,125.4,128.3$, 129.2, 129.4, 129.7, 130.4, 131.8, 132.4, 132.7, 135.5, 137.9, 144.8, 150.4, 157.7, 165.7; MS $m / z 450\left(\mathrm{M}^{+}, 100\right)$;

HRMS calcd for $\mathrm{C}_{24} \mathrm{H}_{22} \mathrm{~N}_{2} \mathrm{O}_{5} \mathrm{~S} 450.1249$, found 450.1242 .

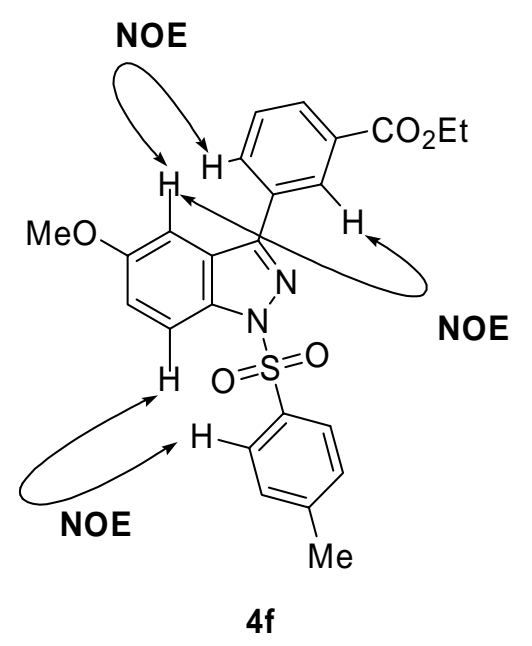

5-Hydroxy-1-(4-methylphenylsulfonyl)-3-phenylindazole (4g-a) (Table 3, Entry 1)<smiles>Oc1ccc2c(c1)c(-c1ccccc1)nn2S</smiles> 
According to the general procedure, a mixture of $3 \mathrm{~g}(50.0 \mathrm{mg}, 0.14 \mathrm{mmol}), \mathrm{Pd}(\mathrm{OAc})_{2}$ (3.1 mg, $0.014 \mathrm{mmol}), \mathrm{Cu}(\mathrm{OAc})_{2}(24.8 \mathrm{mg}, 0.14 \mathrm{mmol})$, and $\mathrm{AgOCOCF}_{3}(60.3 \mathrm{mg}, 0.27$ $\mathrm{mmol})$ in DMSO $(2.7 \mathrm{~mL})$ was stirred for $12 \mathrm{~h}\left(80^{\circ} \mathrm{C}\right.$. $)$ The crude product was purified by silica gel column chromatography [hexane-ethyl acetate (2:1)] to afford $\mathbf{4 g}-\boldsymbol{a}(37.2 \mathrm{mg}$, $75 \%$ ) as a colorless oil.

IR $v$ (film, $\left.\mathrm{cm}^{-1}\right)$ 3481, 1373, 1175, 870, 733, 702, 669;

${ }^{1} \mathrm{H}-\mathrm{NMR}\left(400 \mathrm{MHz}, \mathrm{CDCl}_{3}\right.$ ) d 2.29 (3H, s), 5.47 (1H, br.s), 7.15-7.17 (3H, m), 7.25 (1H, d, $J=2.0 \mathrm{~Hz}), 7.34-7.36(3 \mathrm{H}, \mathrm{m}), 7.74-7.76(2 \mathrm{H}, \mathrm{m}), 7.82(2 \mathrm{H}, \mathrm{d}, J=8.4 \mathrm{~Hz}), 8.06(1 \mathrm{H}, \mathrm{d}, J$ $=8.8 \mathrm{~Hz}$;

${ }^{13} \mathrm{C}-\mathrm{NMR}\left(100 \mathrm{MHz}, \mathrm{CDCl}_{3}\right) \delta 21.6,105.5,114.4,119.6,125.4,127.4,127.9,128.6,129.4$, 129.7, 131.0, 134.1, 136.8, 145.2, 151.6, 153.3;

MS $m / z 364\left(\mathrm{M}^{+}, 98.9\right), 209$ (100), 181 (39.2);

HRMS calcd for $\mathrm{C}_{20} \mathrm{H}_{16} \mathrm{~N}_{2} \mathrm{O}_{3} \mathrm{~S} 364.0881$, found 364.0865 .

\section{5-Amino-1-(4-methylphenylsulfonyl)-3-phenylindazole (4h-a) (Table 3, Entry 2)}<smiles>Nc1ccc2c(c1)c(-c1ccccc1)nn2S</smiles>

According to the general procedure, a mixture of $\mathbf{3 h}(50.0 \mathrm{mg}, 0.14 \mathrm{mmol}), \mathrm{Pd}(\mathrm{OAc})_{2}$ (3.1 mg, $0.014 \mathrm{mmol}), \mathrm{Cu}(\mathrm{OAc})_{2}(24.9 \mathrm{mg}, 0.14 \mathrm{mmol})$, and $\mathrm{AgOCOCF}_{3}(60.4 \mathrm{mg}, 0.27$ mmol) in DMSO $(2.7 \mathrm{~mL})$ was stirred for $18 \mathrm{~h}\left(80^{\circ} \mathrm{C}\right)$. The crude product was purified by silica gel column chromatography [hexane-ethyl acetate (2:1)] to afford $\mathbf{4 h}-\boldsymbol{a}$ (32.9 $\mathrm{mg}$, $66 \%$ ) as a brown oil.

IR $v\left(\right.$ film, $\left.\mathrm{cm}^{-1}\right)$ 3462, 3377, 1626, 1371, 1175, 756, 700, 669;

${ }^{1} \mathrm{H}-\mathrm{NMR}\left(400 \mathrm{MHz}, \mathrm{CDCl}_{3}\right) \delta 2.31(3 \mathrm{H}, \mathrm{s}), 3.81(2 \mathrm{H}$, br.s), $6.96(1 \mathrm{H}, \mathrm{d}, J=8.8 \mathrm{~Hz}), 7.06$ $(1 \mathrm{H}, \mathrm{s}), 7.18(2 \mathrm{H}, \mathrm{d}, J=8.0 \mathrm{~Hz}), 7.42-7.47(3 \mathrm{H}, \mathrm{m}), 7.83(4 \mathrm{H}, \mathrm{m}), 8.01(1 \mathrm{H}, \mathrm{d}, J=8.8 \mathrm{~Hz})$; ${ }^{13} \mathrm{C}-\mathrm{NMR}\left(100 \mathrm{MHz}, \mathrm{CDCl}_{3}\right) \delta 21.6,104.2,114.4,119.3,125.7,127.4,128.0,128.6,129.2$, 129.6, 131.6, 134.4, 136.3, 143.7, 144.8, 151.2;

$\mathrm{MS} m / z 363\left(\mathrm{M}^{+}, 80.0\right), 208(100)$; 
HRMS calcd for $\mathrm{C}_{20} \mathrm{H}_{17} \mathrm{~N}_{3} \mathrm{O}_{2} \mathrm{~S} 363.1041$, found 363.1033.

5-Methoxy-1-(4-methylphenylsulfonyl)-3-phenylindazole

$(4 i-a)^{5)}$

and

3-(3-Methoxyphenyl)-1-(4-methylphenylsulfonyl)indazole (4i-b) (Table 3, Entry 3)<smiles>COc1ccc2c(c1)c(-c1ccccc1)nn2S</smiles>

$4 \mathrm{i}-\mathrm{a}$<smiles>COc1cccc(-c2nn(S)c3ccccc23)c1</smiles>

$4 \mathrm{i}-b$

According to the general procedure, a mixture of $3 \mathbf{i}(50.0 \mathrm{mg}, 0.13 \mathrm{mmol}, \mathrm{A}: \mathrm{B}=1.3: 1)$, $\mathrm{Pd}(\mathrm{OAc})_{2}(2.9 \mathrm{mg}, 0.013 \mathrm{mmol}), \mathrm{Cu}(\mathrm{OAc})_{2}(23.9 \mathrm{mg}, 0.13 \mathrm{mmol})$, and $\mathrm{AgOCOCF}_{3}(58.1$ $\mathrm{mg}, 0.26 \mathrm{mmol})$ in DMSO $(2.6 \mathrm{~mL})$ was stirred for $21 \mathrm{~h}$. The crude product was purified by silica gel column chromatography [hexane-ethyl acetate (4:1)] to afford $\mathbf{4 i - a}(54 \%)$ and $\mathbf{4 i - b}$ (27\%) as an inseparable mixture (40.4 $\mathrm{mg}, 81 \%)$, and $\mathbf{3 i}(1.5 \mathrm{mg}, 3 \%$ as B). The yields of 4i- $\boldsymbol{a}$ and $\mathbf{4 i -} \boldsymbol{b}$ were determined by ${ }^{1} \mathrm{H}-\mathrm{NMR}$ spectrum.

4i-a $\boldsymbol{a}^{5}+\mathbf{4 i}-\boldsymbol{b}$ : IR $v\left(\right.$ film, $\left.\mathrm{cm}^{-1}\right)$ 1377, 1236, 1190, 1177, 669;

${ }^{1} \mathrm{H}-\mathrm{NMR} /{ }^{13} \mathrm{C}-\mathrm{NMR}$ : only a ${ }^{1} \mathrm{H}-\mathrm{NMR}$ spectrum of the mixture of $4 \mathbf{i}-\boldsymbol{a}$ and $\mathbf{4 i}-\boldsymbol{b}$ was obtained;

MS $m / z 378\left(\mathrm{M}^{+}, 94.4\right), 223(100)$;

HRMS calcd for $\mathrm{C}_{21} \mathrm{H}_{18} \mathrm{~N}_{2} \mathrm{O}_{3} \mathrm{~S} 378.1038$, found 378.1020.

Anal. calcd for $\mathrm{C}_{21} \mathrm{H}_{18} \mathrm{~N}_{2} \mathrm{O}_{3} \mathrm{~S}$ : C, 66.65; H, 4.79; N, 7.40. found: C, 66.54; H, 4.93; N, 7.31.

5-Chloro-1-(4-methylphenylsulfonyl)-3-phenylindazole

$(4 \mathrm{j}-a)$

and

3-(3-Chlorophenyl)-1-(4-methylphenylsulfonyl)indazole (4j-b) (Table 3, Entry 4)<smiles>Sn1nc(-c2ccccc2)c2cc(Cl)ccc21</smiles>

$4 \mathrm{j}-\mathrm{a}$<smiles>Sn1nc(-c2cccc(Cl)c2)c2ccccc21</smiles>

$4 j-b$

According to the general procedure, a mixture of $\mathbf{3 j}$ ( $50.0 \mathrm{mg}, 0.13 \mathrm{mmol}$, major isomer : 
minor isomer $=2: 1), \mathrm{Pd}(\mathrm{OAc})_{2}(2.9 \mathrm{mg}, 0.013 \mathrm{mmol}), \mathrm{Cu}(\mathrm{OAc})_{2}(23.6 \mathrm{mg}, 0.13 \mathrm{mmol})$, and $\mathrm{AgOCOCF}_{3}(57.4 \mathrm{mg}, 0.26 \mathrm{mmol})$ in DMSO $(2.6 \mathrm{~mL})$ was stirred for $20 \mathrm{~h}$. The crude product was purified by silica gel column chromatography [hexane-ethyl acetate (9:1)] to afford $\mathbf{4 j - a}(9.4 \mathrm{mg}, 19 \%)$ and $\mathbf{4 j - b}(32.1 \mathrm{mg}, 65 \%)$.

4j-a: $\mathrm{mp} 128-129^{\circ} \mathrm{C}$ (colorless prisms from hexane-ethyl acetate, lit. $\left.{ }^{6}\right) \mathrm{mp} 119-121^{\circ} \mathrm{C}$ );

IR $v\left(\right.$ film, $\left.\mathrm{cm}^{-1}\right) 1385,1192,1175,799,760,677,665$;

${ }^{1} \mathrm{H}-\mathrm{NMR}\left(400 \mathrm{MHz}, \mathrm{CDCl}_{3}\right) \delta 2.36(3 \mathrm{H}, \mathrm{s}), 7.25(2 \mathrm{H}, \mathrm{d}, J=8.0 \mathrm{~Hz}), 7.49-7.55(4 \mathrm{H}, \mathrm{m})$, 7.85-7.90 (5H, m), $8.21(1 \mathrm{H}, \mathrm{d}, J=8.8 \mathrm{~Hz})$;

${ }^{13} \mathrm{C}-\mathrm{NMR}\left(100 \mathrm{MHz}, \mathrm{CDCl}_{3}\right) \delta 21.6,114.7,121.1,125.4,127.7,128.2,128.9,129.6,129.8$, $129.9,130.3,130.9,134.4,140.3,145.5,150.9$;

MS $m / z 384\left(\mathrm{M}^{+}+2,44.8\right), 382\left(\mathrm{M}^{+}, 100\right), 227$ (96.4);

Anal. calcd for $\mathrm{C}_{20} \mathrm{H}_{15} \mathrm{ClN}_{2} \mathrm{O}_{2} \mathrm{~S}: \mathrm{C}, 62.74 ; \mathrm{H}, 3.95 ; \mathrm{N}, 7.32$. found: C, 62.58; H, 4.09; N, 7.27 .

4j-b: $\mathrm{mp} 163-164^{\circ} \mathrm{C}$ (colorless needles from hexane-ethyl acetate);

IR $v\left(\right.$ film, $\left.\mathrm{cm}^{-1}\right)$ 1381, 1190, 1177, 692, 667;

${ }^{1} \mathrm{H}-\mathrm{NMR}\left(400 \mathrm{MHz}, \mathrm{CDCl}_{3}\right) \delta 2.36(3 \mathrm{H}, \mathrm{s}), 7.24(2 \mathrm{H}, \mathrm{d}, J=8.4 \mathrm{~Hz}), 7.39(1 \mathrm{H}, \mathrm{t}, J=7.6$ $\mathrm{Hz}), 7.43-7.44(2 \mathrm{H}, \mathrm{m}), 7.60(1 \mathrm{H}, \mathrm{td}, J=7.8,0.8 \mathrm{~Hz}), 7.79-7.81(1 \mathrm{H}, \mathrm{m}), 7.90-7.92(4 \mathrm{H}$, $\mathrm{m}), 8.28(1 \mathrm{H}, \mathrm{d}, J=8.4 \mathrm{~Hz})$;

${ }^{13} \mathrm{C}-\mathrm{NMR}\left(100 \mathrm{MHz}, \mathrm{CDCl}_{3}\right) \delta 21.7,113.6,121.2,123.8,124.6,126.2,127.5,128.1,129.2$, 129.5, 129.8, 130.0, 133.1, 134.5, 134.7, 141.7, 145.3, 150.0;

MS $m / z 384\left(\mathrm{M}^{+}+2,41.9\right), 382\left(\mathrm{M}^{+}, 100\right), 318$ (72.8), 227 (89.0);

Anal. calcd for $\mathrm{C}_{20} \mathrm{H}_{15} \mathrm{ClN}_{2} \mathrm{O}_{2} \mathrm{~S}: \mathrm{C}, 62.74 ; \mathrm{H}, 3.95 ; \mathrm{N}, 7.32$. found: C, 62.87; H, 3.95; N, 7.33 .

5-Bromo-1-(4-methylphenylsulfonyl)-3-phenylindazole

(4k-a)

and

3-(3-Bromophenyl)-1-(4-methylphenylsulfonyl)indazole (4k-b) (Table 3, Entry 5)<smiles>Sc1nn(S)c2ccc(Br)cc12</smiles>

$4 k-a$<smiles>S=[Y5]1nc(-c2cccc(Br)c2)c2ccccc12</smiles>

$4 k-b$ 
According to the general procedure, a mixture of $3 \mathbf{k}(0.10 \mathrm{~g}, 0.23 \mathrm{mmol}), \mathrm{Pd}(\mathrm{OAc})_{2}$ (5.2 mg, $0.023 \mathrm{mmol}), \mathrm{Cu}(\mathrm{OAc})_{2}(42.3 \mathrm{mg}, 0.23 \mathrm{mmol})$, and $\mathrm{AgOCOCF}_{3}(0.10 \mathrm{~g}, 0.47$ $\mathrm{mmol})$ in DMSO $(4.7 \mathrm{~mL})$ was stirred for $17 \mathrm{~h}$. The crude product was purified by silica gel column chromatography [hexane-ethyl acetate (19:1)] to afford $\mathbf{4 k - \boldsymbol { a }}(33.8 \mathrm{mg}, 34 \%), \mathbf{4 k}-\boldsymbol{b}$ (44.7 mg, 45\%), and 3k (8.8 mg, 9\%).

4k-a: $\mathrm{mp} 109-110^{\circ} \mathrm{C}$ (colorless plates from hexane-ethyl acetate);

IR $v$ (film, $\left.\mathrm{cm}^{-1}\right)$ 1383, 1192, 1175, 673;

${ }^{1} \mathrm{H}-\mathrm{NMR}\left(400 \mathrm{MHz}, \mathrm{CDCl}_{3}\right) \delta 2.36(3 \mathrm{H}, \mathrm{s}), 7.25(2 \mathrm{H}, \mathrm{d}, J=8.2 \mathrm{~Hz}), 7.48-7.54(3 \mathrm{H}, \mathrm{m})$, $7.66(1 \mathrm{H}, \mathrm{dd}, J=9.2,1.4 \mathrm{~Hz}), 7.85(2 \mathrm{H}, \mathrm{dd}, J=7.8,1.8 \mathrm{~Hz}), 7.89(2 \mathrm{H}, \mathrm{d}, J=8.2 \mathrm{~Hz}), 8.05$ $(1 \mathrm{H}, \mathrm{d}, J=1.4 \mathrm{~Hz}), 8.15(1 \mathrm{H}, \mathrm{d}, J=9.2 \mathrm{~Hz})$;

${ }^{13} \mathrm{C}-\mathrm{NMR}\left(100 \mathrm{MHz}, \mathrm{CDCl}_{3}\right) \delta 21.7,114.9,117.7,124.2,125.8,127.5,128.1,128.8,129.7$, $129.8,130.7,132.0,134.2,140.5,145.5,150.7$;

MS $m / z 428\left(\mathrm{M}^{+}+2,100\right), 426\left(\mathrm{M}^{+}, 96.4\right), 364$ (50.2), 362 (50.7), 273 (95.5), 271 (95.5), 163 (98.7);

Anal. calcd for $\mathrm{C}_{20} \mathrm{H}_{15} \mathrm{BrN}_{2} \mathrm{O}_{2} \mathrm{~S}: \mathrm{C}, 56.21 ; \mathrm{H}, 3.54 ; \mathrm{N}, 6.56$. found: C, 56.21; H, 3.49; N, 6.60 .

4k-b: $\mathrm{mp} 159-160^{\circ} \mathrm{C}$ (colorless needles from hexane-ethyl acetate);

IR $v$ (film, $\left.\mathrm{cm}^{-1}\right)$ 1381, 1190, 1177, 750, 685, 667;

${ }^{1} \mathrm{H}-\mathrm{NMR}\left(400 \mathrm{MHz}, \mathrm{CDCl}_{3}\right) \delta 2.35(3 \mathrm{H}, \mathrm{s}), 7.24(2 \mathrm{H}, \mathrm{d}, J=8.0 \mathrm{~Hz}), 7.35-7.40(2 \mathrm{H}, \mathrm{m})$, 7.58-7.61 $(2 \mathrm{H}, \mathrm{m}), 7.84(1 \mathrm{H}, \mathrm{d}, J=8.0 \mathrm{~Hz}), 7.88-7.92(3 \mathrm{H}, \mathrm{m}), 8.06(1 \mathrm{H}, \mathrm{s}), 8.28(1 \mathrm{H}, \mathrm{d}, J$ $=8.4 \mathrm{~Hz})$;

${ }^{13} \mathrm{C}-\mathrm{NMR}\left(100 \mathrm{MHz}, \mathrm{CDCl}_{3}\right) \delta 21.7,113.6,121.2,122.8,123.8,124.5,126.7,127.5,129.2$, 129.8, 130.2, 130.9, 132.4, 133.3, 134.4, 141.7, 145.3, 149.9;

MS $m / z 428\left(\mathrm{M}^{+}+2,100\right), 426\left(\mathrm{M}^{+}, 95.2\right), 364$ (61.3), 362 (61.3), 273 (86.2), 271 (86.7), 163 (77.7);

Anal. calcd for $\mathrm{C}_{20} \mathrm{H}_{15} \mathrm{BrN}_{2} \mathrm{O}_{2} \mathrm{~S}: \mathrm{C}, 56.21 ; \mathrm{H}, 3.54 ; \mathrm{N}, 6.56$. found: C, 56.21; H, 3.71; N, 6.51 . 
1-(4-Methylphenylsulfonyl)-3-(3-nitrophenyl)indazole (4l-b) (Table 3, Entry 6)<smiles>O=[N+]([O-])c1cccc(-c2nn(S)c3ccccc23)c1</smiles>

4I-b

According to the general procedure, a mixture of $3 \mathbf{l}(0.10 \mathrm{~g}, 0.25 \mathrm{mmol}), \mathrm{Pd}(\mathrm{OAc})_{2}(5.7$ $\mathrm{mg}, 0.025 \mathrm{mmol}), \mathrm{Cu}(\mathrm{OAc})_{2}(45.9 \mathrm{mg}, 0.25 \mathrm{mmol})$, and $\mathrm{AgOCOCF}_{3}(0.11 \mathrm{~g}, 0.51 \mathrm{mmol})$ in DMSO $(5.1 \mathrm{~mL})$ was stirred for $24 \mathrm{~h}$. The crude product was purified by silica gel column chromatography [hexane-ethyl acetate (4:1)] to afford $\mathbf{4 l}-\boldsymbol{b}(32.1 \mathrm{mg}, 32 \%)$ and $\mathbf{3 l}(26.0 \mathrm{mg}$, $26 \%)$.

mp $233-234^{\circ} \mathrm{C}$ (colorless prisms from hexane-ethyl acetate);

IR $v\left(\right.$ film, $\left.\mathrm{cm}^{-1}\right) 1375,1348,1178$;

${ }^{1} \mathrm{H}-\mathrm{NMR}\left(400 \mathrm{MHz}, \mathrm{CDCl}_{3}\right) \delta 2.37(3 \mathrm{H}, \mathrm{s}), 7.26-7.28(2 \mathrm{H}, \mathrm{m}), 7.45(1 \mathrm{H}, \mathrm{t}, J=7.7 \mathrm{~Hz})$, $7.64(1 \mathrm{H}, \mathrm{t}, J=7.7 \mathrm{~Hz}), 7.70(1 \mathrm{H}, \mathrm{t}, J=8.3 \mathrm{~Hz}), 7.93(2 \mathrm{H}, \mathrm{d}, J=8.4 \mathrm{~Hz}), 7.94(1 \mathrm{H}, \mathrm{d}, J=$ $8.3 \mathrm{~Hz}), 8.29-8.34(3 \mathrm{H}, \mathrm{m}), 8.76(1 \mathrm{H}, \mathrm{t}, J=2.0 \mathrm{~Hz})$;

${ }^{13} \mathrm{C}-\mathrm{NMR}\left(100 \mathrm{MHz}, \mathrm{CDCl}_{3}\right) \delta 21.7,113.7,120.9,122.9,123.5,124.0,124.9,127.6,129.4$, $129.9,133.2,133.9,134.4,141.8,145.6,148.5,148.8$;

MS $m / z 393\left(\mathrm{M}^{+}, 100\right), 329(73.3)$;

Anal. calcd for $\mathrm{C}_{20} \mathrm{H}_{15} \mathrm{~N}_{3} \mathrm{O}_{4} \mathrm{~S}: \mathrm{C}, 61.06 ; \mathrm{H}, 3.84 ; \mathrm{N}, 10.68$. found: $\mathrm{C}, 61.07 ; \mathrm{H}, 3.86 ; \mathrm{N}$, 10.49 .

1-(4-Methylphenylsulfonyl)-3-(4-nitrophenyl)indazole (4m-b) (Table 3, Entry 7)<smiles>[13CH3]n1nc(-c2ccc([N+](=O)[O-])cc2)c2ccccc21</smiles>

According to the general procedure, a mixture of $3 \mathrm{~m}(50.0 \mathrm{mg}, 0.13 \mathrm{mmol}, E: Z=1$ : 1.2), $\mathrm{Pd}(\mathrm{OAc})_{2}(2.8 \mathrm{mg}, 0.013 \mathrm{mmol}), \mathrm{Cu}(\mathrm{OAc})_{2}(23.0 \mathrm{mg}, 0.13 \mathrm{mmol})$, and $\mathrm{AgOCOCF}_{3}$ 
(55.9 $\mathrm{mg}, 0.25 \mathrm{mmol}$ ) in DMSO $(2.5 \mathrm{~mL})$ was stirred for $24 \mathrm{~h}$. The crude product was purified by silica gel column chromatography [hexane-ethyl acetate $(4: 1)$ ] to afford $\mathbf{4 m}-\boldsymbol{b}$ (13.2 mg, 27\%) and 3m (13.6 mg, 27\%, E: $Z=1.2: 1$ ).

$\mathrm{mp} 237-238^{\circ} \mathrm{C}$ (light yellow prisms from ethyl acetate);

IR $v$ (film, $\left.\mathrm{cm}^{-1}\right)$ 1522, 1348, 1192, 681;

${ }^{1} \mathrm{H}-\mathrm{NMR}\left(400 \mathrm{MHz}, \mathrm{CDCl}_{3}\right) \delta 2.37(3 \mathrm{H}, \mathrm{s}), 7.27(2 \mathrm{H}, \mathrm{d}, J=8.4 \mathrm{~Hz}), 7.44(1 \mathrm{H}, \mathrm{t}, J=8.2$ $\mathrm{Hz}), 7.63(1 \mathrm{H}, \mathrm{t}, J=8.2 \mathrm{~Hz}), 7.92(1 \mathrm{H}, \mathrm{d}, J=8.2 \mathrm{~Hz}), 7.93(2 \mathrm{H}, \mathrm{d}, J=8.4 \mathrm{~Hz}), 8.12(2 \mathrm{H}, \mathrm{d}$, $J=9.0 \mathrm{~Hz}), 8.31(1 \mathrm{H}, \mathrm{d}, J=8.2 \mathrm{~Hz}), 8.36(2 \mathrm{H}, \mathrm{d}, J=9.0 \mathrm{~Hz})$;

${ }^{13} \mathrm{C}-\mathrm{NMR}\left(100 \mathrm{MHz}, \mathrm{CDCl}_{3}\right) \delta 21.7,113.7,121.0,123.5,124.0,124.9,127.6,128.8,129.4$, 129.8, 134.3, 137.7, 141.7, 145.6, 148.1, 148.7;

MS $m / z 393\left(\mathrm{M}^{+}, 100\right), 329$ (59.5), 155 (63.8);

HRMS calcd for $\mathrm{C}_{20} \mathrm{H}_{15} \mathrm{~N}_{3} \mathrm{O}_{4} \mathrm{~S}$ 393.0783, found 393.0786.

6-Methoxy-1-(4-methylphenylsulfonyl)-3-phenylindazole

(4n-a)

and 3-(4-Methoxyphenyl)-1-(4-methylphenylsulfonyl)indazole (4n-b) (Table 3, Entry 8)<smiles>COc1ccc2c(-c3ccccc3)nn(S)c2c1</smiles>

$4 n-a$<smiles>COc1ccc(-c2nn(S)c3ccccc23)cc1</smiles>

$4 n-b$

According to the general procedure, a mixture of $3 \mathrm{n}(50.0 \mathrm{mg}, 0.13 \mathrm{mmol}, E: Z=1$ : 0.04), $\mathrm{Pd}(\mathrm{OAc})_{2}(2.9 \mathrm{mg}, 0.013 \mathrm{mmol}), \mathrm{Cu}(\mathrm{OAc})_{2}(23.9 \mathrm{mg}, 0.13 \mathrm{mmol})$, and $\mathrm{AgOCOCF}_{3}$ $(58.1 \mathrm{mg}, 0.26 \mathrm{mmol})$ in DMSO $(2.6 \mathrm{~mL})$ was stirred for $24 \mathrm{~h}$. The crude product was purified by silica gel column chromatography [hexane-ethyl acetate $(4: 1)$ ] to afford $4 \mathbf{n}-\boldsymbol{a}$ (3.0 mg, 6\%), 4n-b (8.0 mg, 16\%), and 3n (28.3 mg, 57\%, E: Z=1:1).

4n-a: $\mathrm{mp} 155-156^{\circ} \mathrm{C}$ (colorless plates from hexane-ethyl acetate);

IR $v$ (film, $\left.\mathrm{cm}^{-1}\right)$ 1614, 1366, 1173, 671;

${ }^{1} \mathrm{H}-\mathrm{NMR}\left(400 \mathrm{MHz}, \mathrm{CDCl}_{3}\right) \delta 2.35(3 \mathrm{H}, \mathrm{s}), 3.97(3 \mathrm{H}, \mathrm{s}), 6.97(1 \mathrm{H}, \mathrm{dd}, J=9.0,2.4 \mathrm{~Hz})$, $7.23(2 \mathrm{H}, \mathrm{d}, J=8.2 \mathrm{~Hz}), 7.44-7.52(3 \mathrm{H}, \mathrm{m}), 7.69(1 \mathrm{H}, \mathrm{d}, J=2.4 \mathrm{~Hz}), 7.75(1 \mathrm{H}, \mathrm{d}, J=9.0$ 
$\mathrm{Hz}), 7.85-7.90$ (4H, m);

${ }^{13} \mathrm{C}-\mathrm{NMR}\left(100 \mathrm{MHz}, \mathrm{CDCl}_{3}\right) \delta 21.6,55.8,95.6,115.5,118.5,122.3,127.6,128.2,128.8$, $129.5,129.8,131.5,134.7,143.7,145.2,151.6,161.1$;

MS $m / z 378\left(\mathrm{M}^{+}, 62.9\right), 223$ (100), 195 (56.5);

HRMS calcd for $\mathrm{C}_{21} \mathrm{H}_{18} \mathrm{~N}_{2} \mathrm{O}_{3} \mathrm{~S} 378.1039$, found 378.1016 .

4n-b: $143-145^{\circ} \mathrm{C}$ (colorless plates from hexane-ethyl acetate, lit. ${ }^{5)} \mathrm{mp} 143-145^{\circ} \mathrm{C}$ );

IR $v$ (film, $\left.\mathrm{cm}^{-1}\right)$ 1371, 1256, 1177;

${ }^{1} \mathrm{H}-\mathrm{NMR}\left(400 \mathrm{MHz}, \mathrm{CDCl}_{3}\right) \delta 2.31(3 \mathrm{H}, \mathrm{s}), 3.85(3 \mathrm{H}, \mathrm{s}), 7.01(2 \mathrm{H}, \mathrm{d}, J=8.8 \mathrm{~Hz}), 7.20(2 \mathrm{H}$, $\mathrm{d}, J=8.0 \mathrm{~Hz}), 7.34(1 \mathrm{H}, \mathrm{t}, J=8.0 \mathrm{~Hz}), 7.55(1 \mathrm{H}, \mathrm{t}, J=8.0 \mathrm{~Hz}), 7.84-7.89(5 \mathrm{H}, \mathrm{m}), 8.24$ $(1 \mathrm{H}, \mathrm{d}, J=8.0 \mathrm{~Hz})$;

${ }^{13} \mathrm{C}-\mathrm{NMR}\left(100 \mathrm{MHz}, \mathrm{CDCl}_{3}\right) \delta 21.6,55.4,113.5,114.2,121.6,123.9,124.2,124.3,127.5$, $128.9,129.5,129.6,134.6,141.8,145.0,151.4,160.6$;

$\mathrm{MS} m / z 378\left(\mathrm{M}^{+}, 75.2\right), 223(100)$;

HRMS calcd for $\mathrm{C}_{21} \mathrm{H}_{18} \mathrm{~N}_{2} \mathrm{O}_{3} \mathrm{~S} 378.1038$, found 378.1043.

6-Methoxy-1-(4-methylphenylsulfonyl)-3-phenylindazole

(4n-a)

and

3-(4-Methoxyphenyl)-1-(4-methylphenylsulfonyl)indazole (4n-b) (Table 3, Entry 9)<smiles>COc1ccc2c(-c3ccccc3)nn(S)c2c1</smiles>

$4 n-a$<smiles>COc1ccc(-c2nn(S)c3ccccc23)cc1</smiles>

$4 n-b$

According to the general procedure, a mixture of $3 \mathbf{n}(40.0 \mathrm{mg}, 0.11 \mathrm{mmol}, E: Z=0.31$ : 1), $\mathrm{Pd}(\mathrm{OAc})_{2}(2.4 \mathrm{mg}, 0.011 \mathrm{mmol}), \mathrm{Cu}(\mathrm{OAc})_{2}(19.1 \mathrm{mg}, 0.11 \mathrm{mmol})$, and $\mathrm{AgOCOCF}_{3}$ (46.4 mg, $0.21 \mathrm{mmol})$ in DMSO $(2.1 \mathrm{~mL})$ was stirred for $24 \mathrm{~h}$. The crude product was purified by silica gel column chromatography [hexane-ethyl acetate (4:1)] to afford $4 \mathbf{n}-\boldsymbol{a}$ (5.5 mg, 14\%), 4n-b (6.9 mg, 17\%), and 3n (20.6 mg, 52\%, $E: Z=1: 1.3)$. 
1-(4-Methylphenylsulfonyl)-3-phenylindazole (4o-c=2) (Table 3, Entry 10)<smiles>[As]n1nc(-c2ccccc2)c2ccccc21</smiles>

According to the general procedure, a mixture of $3 \mathbf{o}(0.10 \mathrm{~g}, 0.23 \mathrm{mmol}), \operatorname{Pd}(\mathrm{OAc})_{2}(5.2$ $\mathrm{mg}, 0.023 \mathrm{mmol}), \mathrm{Cu}(\mathrm{OAc})_{2}$ (42.3 mg, $\left.0.23 \mathrm{mmol}\right)$, and $\mathrm{Ag}\left(\mathrm{OCOCF}_{3}\right)(0.10 \mathrm{~g}, 0.47 \mathrm{mmol})$ in DMSO $(4.7 \mathrm{~mL})$ was stirred for $14 \mathrm{~h}$. The crude product was purified by silica gel column chromatography [hexane-ethyl acetate $(4: 1)$ ] to afford $2(79.8 \mathrm{mg}, 98 \%)$ as a colorless solid.

\section{Synthesis of 3-Alkylindazole (Scheme 1)}

\section{2,2-Dimethylpropiophenone 4-Methylphenylsulfonylhydrazone (5)}<smiles>CN=C(N=N[Mg][Mg])c1ccccc1</smiles>

Acetyl chloride $(0.15 \mathrm{~g}, 1.9 \mathrm{mmol})$ was slowly added to ethanol $(1 \mathrm{~mL})$ at $0^{\circ} \mathrm{C}$ and stirred for $30 \mathrm{~min}$ at the same temperature. The reaction mixture was warmed to room temperature and stirred for another $30 \mathrm{~min}$. The resulting mixture and a solution of the 2,2-dimethylpropiophenone $(62.1 \mathrm{mg}, 0.38 \mathrm{mmol})$ in ethanol $(2 \mathrm{~mL})$ were added to a solution of 4-methylphenylsulfonylhydrazide $(0.14 \mathrm{~g}, 0.77 \mathrm{mmol})$ in ethanol $(2 \mathrm{~mL})$ and heated under reflux for $2 \mathrm{~h}$. The mixture was treated with saturated aqueous $\mathrm{NaHCO}_{3}(5$ $\mathrm{mL})$ and extracted with ethyl acetate $(10 \mathrm{~mL} \times 3)$. The combined organic layer was washed with brine $(10 \mathrm{~mL})$ and dried over $\mathrm{MgSO}_{4}$. The solvent was evaporated and the residue was purified by silica gel column chromatography [hexane-ethyl acetate (9:1)] to afford 2,2-dimethylpropiophenone 4-methylphenylsulfonylhydrazone 5 (99.5 mg, 79\%, single isomer) as a colorless solid.

$\mathrm{mp} 159-160^{\circ} \mathrm{C}$ (colorless needles from hexane-ethyl acetate, lit. ${ }^{6}{ }^{\mathrm{mp}} 156-158^{\circ} \mathrm{C}$ ); IR $v$ (film, $\left.\mathrm{cm}^{-1}\right) 3223,1169$;

${ }^{1} \mathrm{H}-\mathrm{NMR}\left(400 \mathrm{MHz}, \mathrm{CDCl}_{3}\right) \delta 1.06(9 \mathrm{H}, \mathrm{s}), 2.45(3 \mathrm{H}, \mathrm{s}), 6.80-6.82(2 \mathrm{H}, \mathrm{m}), 6.88(1 \mathrm{H}, \mathrm{s})$, 
$7.33(2 \mathrm{H}, \mathrm{d}, J=8.4 \mathrm{~Hz}), 7.40-7.41(3 \mathrm{H}, \mathrm{m}), 7.77(2 \mathrm{H}, \mathrm{d}, J=8.4 \mathrm{~Hz})$;

${ }^{13} \mathrm{C}-\mathrm{NMR}\left(100 \mathrm{MHz}, \mathrm{CDCl}_{3}\right) \delta 21.5,27.9,38.4,127.3,127.8,129.1,129.2,131.6,135.3$, 143.8, 165.4;

MS $m / z 330\left(\mathrm{M}^{+}, 8.9\right), 119(100)$;

HRMS calcd for $\mathrm{C}_{18} \mathrm{H}_{22} \mathrm{~N}_{2} \mathrm{O}_{2} \mathrm{~S} 330.1402$, found 330.1395 .

\section{3-tert-Butyl-1-(4-methylphenylsulfonyl)indazole}<smiles>Sn1nc(Br)c2ccccc21</smiles>

A mixture of 2,2-dimethylpropiophenone 4-methylphenylsulfonylhydrazone (50 $\mathrm{mg}$, $0.15 \mathrm{mmol}), \mathrm{Pd}(\mathrm{OAc})_{2}(3.4 \mathrm{mg}, 0.015 \mathrm{mmol}), \mathrm{Cu}(\mathrm{OAc})_{2}(27.5 \mathrm{mg}, 0.15 \mathrm{mmol})$, and $\mathrm{AgOCOCF}_{3}(66.8 \mathrm{mg}, 0.30 \mathrm{mmol})$ in DMSO $(3.0 \mathrm{~mL})$ was stirred at $50^{\circ} \mathrm{C}$ for $24 \mathrm{~h}$. The reaction mixture was extracted with ethyl acetate $(5 \mathrm{~mL} \times 3)$ and the combined organic layer was washed with brine $(10 \mathrm{~mL})$, and dried over $\mathrm{MgSO}_{4}$. The solvent was evaporated and the residue was purified by silica gel column chromatography [hexane-ethyl acetate (9:1)] to afford 3-tert-butyl-1-(4-methylphenylsulfonyl)indazole 6 (32.9 mg, 66\%), and hydrazone 5 (16.0 mg, 32\%).

$\mathrm{mp} 146-147^{\circ} \mathrm{C}$ (colorless prisms from hexane-ethyl acetate);

IR $v$ (film, $\left.\mathrm{cm}^{-1}\right) 1377,1177,712,665$;

${ }^{1} \mathrm{H}-\mathrm{NMR}\left(400 \mathrm{MHz}, \mathrm{CDCl}_{3}\right) \delta 1.45(9 \mathrm{H}, \mathrm{s}), 2.33(3 \mathrm{H}, \mathrm{s}), 7.19(2 \mathrm{H}, \mathrm{d}, J=8.0 \mathrm{~Hz}), 7.26(1 \mathrm{H}$, ddd, $J=8.1,7.2,0.8 \mathrm{~Hz}), 7.48(1 \mathrm{H}, \mathrm{ddd}, J=8.4,7.2,1.2 \mathrm{~Hz}), 7.78-7.82(3 \mathrm{H}, \mathrm{m}), 8.18(1 \mathrm{H}$, dd, $J=8.4,0.8 \mathrm{~Hz})$;

${ }^{13} \mathrm{C}-\mathrm{NMR}\left(100 \mathrm{MHz}, \mathrm{CDCl}_{3}\right) \delta 21.6,29.4,34.3,113.7,122.3,123.3,124.2,127.4,128.3$, 129.4, 134.4, 142.1, 144.7, 161.1;

MS $m / z 328\left(\mathrm{M}^{+}, 74.1\right), 313(100)$;

HRMS calcd for $\mathrm{C}_{18} \mathrm{H}_{20} \mathrm{~N}_{2} \mathrm{O}_{2} \mathrm{~S} 328.1246$, found 328.1232;

Anal. calcd for $\mathrm{C}_{18} \mathrm{H}_{20} \mathrm{~N}_{2} \mathrm{O}_{2} \mathrm{~S}$ : C, 65.83; H, 6.14; N, 8.53. found: C, 65.83; H, 6.17; N, 8.55.

\section{References}

1) Sacks, C. E.; Fuchs, P. L. Synthesis 1976, 456-457. 
2) Bamford, W. R.; Stevens, T. S. J. Chem. Soc. 1952, 4735-4740.

3) Joshi, V.; Sharma, R. K. J. Indian Chem. Soc. 1988, 65, 564-566.

4) Humphreys, R. W. R.; Arnold, D. R. Can. J. Chem. 1979, 57, 2652-2661.

5) Inamoto, K.; Katsuno, M.; Yoshino, T.; Arai, Y.; Hiroya, K.; Sakamoto, T. Tetrahedron 2007, 63, 2695-2711.

6) Knollmueller, M.; Fauss, R. Monatshefte fuer Chemie 1985, 116, 1027-1040.

7) Celebi, S.; Leyva, S.; Modarelli, D. A.; Platz, M. S. J. Am. Chem. Soc. 1993, 115, 8613-8620. 